\title{
SUMOylation promotes de novo targeting of HP1a to pericentric heterochromatin
}

Christèle Maison ${ }^{1}$, Delphine Bailly ${ }^{1}$, Danièle Roche ${ }^{1}$, Rocío Montes de Oca ${ }^{1}$, Aline V. Probst ${ }^{1 \#}$, Isabelle Vassias $^{1}$, Florent Dingli ${ }^{2}$, Bérengère Lombard ${ }^{2}$, Damarys Loew ${ }^{2}$, Jean-Pierre Quivy ${ }^{1} *$ and Geneviève Almouzni ${ }^{1 *}$

${ }^{1}$ Institut Curie UMR 218 CNRS, Laboratory of Nuclear Dynamics and Genome Plasticity, 26 rue d'Ulm, 75248 Paris Cedex 05, France

${ }^{2}$ Institut Curie, Laboratory of Proteomic Mass Spectrometry, 26 rue d'Ulm, 75248 Paris Cedex 05, France

\# Present address: CNRS, UMR 6247 GreD, Clermont Université, INSERM U931, 24 avenue des Landais, 63177 Aubière, France

* Corresponding authors

For correspondence: G. Almouzni

Tel: + $33(0) 156246701$

Fax: + $33(0) 146333016$

E-mail: genevieve.almouzni@curie.fr

Keywords: HP1 $\alpha$, sumoylation, non-coding centromeric RNAs, pericentric heterochromatin 


\begin{abstract}
HP1 enrichment at pericentric heterochromatin is considered important for centromere function. While HP1 binding to $\mathrm{H} 3 \mathrm{~K} 9 \mathrm{me} 3$ can explain its accumulation at pericentric heterochromatin, how it is initially targeted there remains unclear. Here, in mouse cells, we reveal the presence of long nuclear non-coding transcripts corresponding to major satellite repeats at the periphery of pericentric heterochromatin. Furthermore, we find that major transcripts in the forward orientation specifically associate with SUMOmodified HP1 proteins. We identified this modification as SUMO-1 and mapped it in the hinge domain of HP1 $\alpha$. Importantly, the hinge domain and its sumoylation prove critical to promote the initial targeting of HP1 $\alpha$ to pericentric domains using de novo localization assays whereas they are dispensable for maintenance of HP1 domains. We propose that SUMO-HP1, through a specific association with major forward transcript, is guided at pericentric heterochromatin domain to seed further HP1 localization.
\end{abstract}


Heterochromatin at pericentric domains represents a paradigm for understanding how a functional nuclear domain is established and maintained. Studies in S. pombe and Drosophila have advanced our knowledge concerning basic mechanisms and conserved components in the organization of the domain ${ }^{1}$. In particular HP1 proteins which accumulate in these domains are highly conserved from S. pombe to mammals. However in mammalian cells, how HP1 is specifically targeted de novo to initiate formation of a domain of accumulation as found in pericentric heterochromatin ${ }^{2,3}$ remains mysterious. The recognition of $\mathrm{H} 3 \mathrm{~K} 9 \mathrm{me} 3$ by $\mathrm{HP} 1^{4,5}$ which exemplifies the paradigm of the reader model in the histone code hypothesis ${ }^{6,7}$ does not necessarily provide on its own a de novo specific targeting mechanism. One should also consider other HP1 binding partners ${ }^{8,9}$, potential post-translational modifications ${ }^{10}$ and the elusive RNA that has been linked to the presence of HP1 at pericentric heterochromatin ${ }^{11,12}$. While transcripts from major satellite DNA repeats have been identified ${ }^{13,14}$, a functional connection between specific RNAs and HP1 has not yet been established. Taken together, these data prompted us to explore further the HP1-RNA connection and particular post-translational modifications or partners that could provide a means for the de novo targeting of HP1 to pericentric heterochromatin, thereby helping to define this specific subnuclear compartment.

We first verified that repetitive DNA sequences in mouse centromeres, known as major and minor satellites in pericentric and centric heterochromatin respectively ${ }^{15}$, can be transcribed in both orientations. All these transcripts detected by RT-PCR analysis with strand-specific primers for major or minor satellites showed various sizes corresponding to multiple repeats of their basic units (Fig. 1a). We next examined whether some of these transcripts could be stably found in the nucleus. As a reference for comparison, we used the well-defined organization of chromocenters with major satellite repeats surrounded by minor satellite DNA (Fig. 1b, left, DNA FISH) ${ }^{2}$. For this, we exploited fluorescently labelled locked nucleic acid (LNA) probes for RNA FISH that detect specifically major and minor transcripts in either forward or reverse orientation. While minor RNAs were barely detectable, LNA probes for major RNAs in both orientations revealed a significant number of nuclear spots frequently associated or close to chromocenters (Fig. 1b, middle). These signals were not a result of DNA cross-hybridization, since they were undetectable after RNase treatment (Supplementary Fig. 1). With some variations from cell to cell suggesting cell cycle modulation, reverse major RNAs were usually detected as fewer and larger spots (Fig. 1b, middle, red) and forward major RNA signals were smaller in size and present in higher numbers (green). Interestingly, these forward major RNAs were consistently at the periphery of HP1 $\alpha$ domains (Fig. 1b, right, immuno-RNA FISH and Supplementary Fig. 2a). Since active transcription occurs frequently in the external part of chromosomal domains by DNA looping out ${ }^{16}$, they may represent primary transcripts stably maintained at the site of transcription. Notably, HP1 $\alpha$ accumulated at major satellite domains, away from minor satellites 
which were juxtaposed (Fig. 1c and Supplementary Fig. 2b, Immuno-DNA FISH) ${ }^{2}$. We then examined further the possible in vivo association of major RNAs with major satellite domains by other methods. Under conditions that preserved RNAs, we immunoprecipitated heterochromatin-associated material with a specific HP1 $\alpha$ antibody. We found a significantly higher amount of forward major transcripts compared to reverse transcripts (Fig. 1d). Taken together, our data highlight a particular link between major RNA in the forward orientation and pericentric heterochromatin. While HP1 $\alpha$ can bind RNA in vitro ${ }^{12}$, a specific binding to a given transcript has been reported so far only for TERRA RNA ${ }^{17}$. Using GST-tagged HP1 $\alpha$ full-length protein or fragments thereof and radioactively labelled centromeric RNA probes (Fig. 2a), we find that the hinge domain $(\mathrm{H})$ of HP1 $\alpha$ strongly recognized both forward and reverse centromeric RNA probes, while the chromo-hinge $(\mathrm{CD}+\mathrm{H})$ and the hinge-chromoshadow $(\mathrm{H}+\mathrm{CSD})$ domains showed a lower binding capacity. Under these conditions, we did not detect binding between full-length HP1 $\alpha$ and RNAs (Fig. 2a). These results suggest that the central hinge domain may adopt a different conformation in the full-length protein, possibly constrained by the CD and/or the CSD. This raised the possibility that HP1 $\alpha$ could actually bind RNA in vivo and that this interaction can be regulated. To investigate this hypothesis, we aimed to identify proteins from nuclear cell extracts that associated with centromeric RNAs, using in vitro transcribed biotin-labelled forward major or minor RNAs immobilized on streptavidin beads (Fig. 2b and Supplementary Fig. 3a). Mass spectrometry analysis of the RNA-associated proteins identified mainly hnRNPs and proteins involved in RNA processing (Supplementary Fig. 3b and Supplementary Table 1) including vigilin, RNA helicase A (RHA) and Ras-GAP SH3 domain binding protein (G3bp), which were all validated by Western blotting (Fig. 2c). Vigilin and the Drosophila RHA ortholog, maleless (MLE), are thought to play roles in heterochromatin formation and X-chromosome dosage compensation, respectively ${ }^{18,19}$, while G3bp may be involved in RNA metabolism ${ }^{20}$. Surprisingly, under these experimental conditions, we did not detect any significant association between HP1 $\alpha$ and major or minor RNAs, neither by mass spectrometry (Supplementary Fig. 3b) nor by Western blotting (Fig. 2c). Given our in vitro observations (Fig. 2a), we wondered whether post-translational modification of HP1 $\alpha$ could promote in vivo HP1 $\alpha$ binding to RNA. Indeed, HP1 can be phosphorylated ${ }^{10}$ and in fission yeast, Swi6/HP1 is sumoylated in vivo ${ }^{21}$. Furthermore, defective sumoylation of Swi6 results in significantly reduced heterochromatin stability. This prompted us to repeat our RNA pull down strategy with nuclear extracts prepared in the presence of a cocktail of phosphatase inhibitors and N-ethylmaleimide (NEM), a strong inhibitor of SUMO isopeptidases ${ }^{22}$. Remarkably, Western blotting with an anti-HP1 $\alpha$ antibody revealed a slower migrating band in the input for HP1 $\alpha$ that was not detected in absence of NEM and that then was specifically enriched in precipitates with forward major RNAs corresponding to either one or two satellite repeats length (Fig. 2d; Maj1 F or Maj2 F). This band, with an approximate additional molecular mass of $\sim 11 \mathrm{kDa}$ compared to HP1 $\alpha$, might represent an ubiquitin or a SUMO moiety. We confirmed that 
this slower-migrating form was modified by SUMO-1, but not SUMO-2/3, using specific antibodies (Fig. 2d). We obtained similar results with antibodies specific of HP1 $\beta$ and HP1 $\gamma$ (Supplementary Fig. 3c) indicating that all HP1 isoforms can potentially be sumoylated in vivo and are specifically retrieved with forward major RNAs. While the forward major RNA pulls down SUMO-1-HP1 $\alpha$, we estimated that only a small percentage of HP1 $\alpha$ was SUMO-1 modified in vivo (less than $1 \%$ of total protein from nuclear extracts, Fig. 2d). This low representation is in agreement with rapid cycles of sumoylation and desumoylation as reported for other proteins weakly detected in a SUMO-modified state in vivo ${ }^{23,24}$.

To confirm independently that HP1 $\alpha$ gets sumoylated in vivo, we co-transfected HA-tagged HP1 $\alpha$ (e-HP1 $\alpha$ ) and GFP-SUMO-1 into NIH3T3 cells to prepare total cell extracts and carried out immunoprecipitations with anti-HA beads under conditions that preserved the SUMO modification ${ }^{25}$. Western blot analysis using anti-GFP antibodies clearly revealed a band corresponding to GFP-SUMO-eHP1 $\alpha$ (Supplementary Fig. 4a), demonstrating unambiguously that HP1 $\alpha$ can be sumoylated in vivo. To determine which region of HP1 $\alpha$ was SUMO-modified, we next used an in vitro sumoylation assay with either wildtype (W) or mutant (M) SUMO-1 protein, in the presence of E1 activating and E2 conjugating (Ubc9) SUMO enzymes and various GST-HP1 $\alpha$ domains (Fig. 3a). Western blot analysis using anti-GST antibodies showed SUMO-modification on the full-length HP1 $\alpha$, the chromo-hinge, the hingechromoshadow and the hinge domains, the latter domain showing the highest level of sumoylation (Fig. $3 \mathrm{~b}$ and Supplementary Fig. 4b, asterisks). This is also true when using SUMO-2 or SUMO-3 (Supplementary Fig. 4c). These results revealed that the hinge, the same domain of HP1 $\alpha$ that displayed RNA binding activity (Fig. 2a), is a target for sumoylation. Since the fusion of Ubc9 to a substrate provides a convenient way to increase its sumoylation ${ }^{26}$, we also verified with a GST-HP1 $\alpha$-Ubc9 fusion protein that we could enhance SUMO-1-modification of HP1 $\alpha$ in vitro without adding the E2 enzyme (Fig. 3c). Then, using the GST-HP1 $\alpha$ hinge and SUMO-1 proteins as above (Supplementary Fig. 5a), we further identified specific sumoylated residues on HP1 $\alpha$ by mass spectrometry. In the hinge domain, among the 13 lysines $(\mathrm{K})$ as potential targets for sumoylation, we found that K84 of HP1 $\alpha$ in the peptide, "EKSEGNK", was unequivocally identified as sumoylated by mass (with high accuracy in the orbitrap; Fig. 3d, right spectra, arrow) and sequence (by MS/MS in the Qstar mass spectrometer; Fig. 3d; shown y

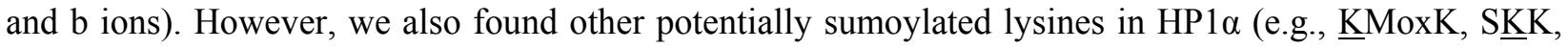
$\underline{K} Y K$ or YKKK; Supplementary Fig. 5b and c) indicating alternative usage of various lysine residues. We thus mutated successively each of the individual 13 lysines to arginine and performed in vitro sumoylation assays as above and in vivo co-transfections as in Supplementary Figure 4a. We observed in all cases that once we mutated one residue an alternative sumoylation site was used (data not shown), showing the 
usage of more than one sumoylation site and corroborating our mass spectrometry data. This suggests a lack of a strict requirement for a "specific" SUMO modified residue.

Since the fusion of Ubc9 to HP1 $\alpha$ increases sumoylation efficiency in vitro (Fig. 3c), we thus generated an HP1 $\alpha$-Ubc9-HA fusion construct (e-HP1 $\alpha$-Ubc9) for in vivo expression and further analysis in a cellular context. Transfection of e-HP1 $\alpha$-Ubc9 or e-HP1 $\alpha$ into NIH3T3 cells lead to comparable levels of proteins being expressed (Supplementary Fig. 6a and b; anti-HA). Interestingly, a significantly higher amount of SUMO-modified e-HP1 $\alpha-\mathrm{Ubc} 9$ could be detected. In total cell extracts, we found that eHP1 $\alpha$-Ubc9 was mainly SUMO-2/3-modified reflecting the readily available endogenous SUMO-2/3 proteins compared to the limited amount of free endogenous SUMO-1 protein in cells (compare SUMO-1 vs SUMO-2/3 inputs in Fig. 2d $)^{23}$. This could be compensated for by providing exogenous SUMO-1 by transient transfection (Supplementary Fig. 6c). These in vivo results underline the fact that HP1 $\alpha$ can be modified by SUMO-1 or SUMO-2/3 depending on the available substrate, as shown in vitro (Fig. 3b\&c and Supplementary Fig. 4b\&c). We verified that the catalytic activity of the fused Ubc9 was directly involved in HP1 $\alpha$ sumoylation enhancement using a Ubc9 catalytic mutant fused to HP1 $\alpha$ (e-HP1 $\alpha$ Ubc9C93S). In comparaison with e-HP1 $\alpha-\mathrm{Ubc} 9 \mathrm{wt}$, we detected a strongly reduced sumoylation of eHP1 $\alpha$-Ubc9C93S (Supplementary Fig. 6c). Next, we examined e-HP1 $\alpha$-Ubc9 and e-HP1 $\alpha$ localization in NIH3T3 cells. Both of them could accumulate at pericentric domains where endogenous HP1 $\alpha$ is already located (Fig. 3e). Thus under these conditions, promoting HP1 sumoylation did not give a particular advantage for the recruitment and maintenance of exogenous HP1 to preexisting HP1 domains of accumulation. Next, we wondered whether HP1 $\alpha$ sumoylation could be required more specifically for a de novo targeting of HP1 $\alpha$ to heterochromatin domains. To test this hypothesis we used MEFs derived from Suv39h double-null (dn) mice, in which the H3K9me3 mark and HP1 $\alpha$ are no longer enriched at pericentric heterochromatin $^{11,27}$ (Fig. 4). Transfection with exogenous Myc-SUV39H1 can restore the proper localization of these marks (Fig. 4b) 5,28 $^{5}$ Since we postulated that the interaction between major RNAs and sumoylated HP1 $\alpha$ targets HP1 $\alpha$ to pericentric domains, we first verified that we could detect major RNAs by RNA-FISH in Suv39h dn MEFs (Supplementary Fig. 7a). We then transiently transfected Suv39h dn cells with e-HP1 $\alpha$ or e-HP1 $\alpha$-Ubc9 in the absence of Myc-SUV39H1 and verified that the proteins were expressed at comparable levels (Fig. 4c). We could not detect the typical localization to chromocenters in cells transfected with e-HP1 $\alpha$ (Fig. 4c). In contrast, in about $10 \%$ of cells transfected with e-HP1 $\alpha$-Ubc9 cells, we found a faint but detectable pericentric localization $6 \mathrm{~h}$ post-transfection. Remarkably, in the latter case, we could not detect $\mathrm{H} 3 \mathrm{~K} 9 \mathrm{me} 3$ accumulation at pericentric heterochromatin (Fig. 4c and Supplementary Fig. 7b) while H3K9me1 was clearly visible at these domains in all Suv39h dn cells (Supplementary Fig. 7c). Thus, a targeting of e-HP1 $\alpha-\mathrm{Ubc} 9$ to pericentric heterochromatin could 
occur in the absence of SUV39H1-dependent H3K9me3. However, while e-HP1 $\alpha$-Ubc9 was more efficiently targeted than e-HP1 $\alpha$, the low fraction of cells showing this staining suggested that following this initial recruitment, retention at pericentric domains was rather inefficient. We thus modified our assay to monitor the localization of e-HP1 $\alpha$ or e-HP1 $\alpha$-Ubc9 to pericentric chromatin in the presence of MycSUV39H1 assuming that H3K9me3 could promote stabilization (Fig. 4d). Immunofluorescence analysis revealed that e-HP1 $\alpha$, e-HP1 $\alpha$-Ubc9C93S and e-HP1 $\alpha$-Ubc9wt accumulated at pericentric heterochromatin when co-transfected with Myc-SUV39H1, in contrast to a negative control protein ehnRNPC (Fig. 4e). Remarkably, e-HP1 $\alpha$-Ubc9wt localized to these domains more efficiently than e-HP1 $\alpha$ and e-HP1 $\alpha$-Ubc9C93S (62\% vs 37\% and 39\% of positive cells, respectively $6 \mathrm{~h}$ post-transfection) for comparable levels of expressed proteins (Supplementary Fig. 8a). Also, the e-HP1 $\alpha$ and e-HP1 $\alpha-$ Ubc9C93S overall staining in the nucleus was rather diffuse compared to e-HP1 $\alpha$-Ubc9wt staining suggesting that a significant fraction of e-HP1 $\alpha$ and e-HP1 $\alpha$-Ubc9C93S is not localized at pericentric heterochromatin (Fig. 4e, Supplementary Fig. 8b and supplemental legend for details). Taken together, these results indicate that enhancing sumoylation on HP1 $\alpha$ promotes a more efficient accumulation at pericentric heterochromatin. We then performed a time course analysis to compare the appearance of HP1 $\alpha$ at pericentric heterochromatin, in Suv39h dn cells co-transfected with Myc-SUV39H1 and e-HP1 $\alpha$ or e-HP1 $\alpha$-Ubc9 (Fig. 4f). We found that e-HP1 $\alpha$-Ubc9 always localized more efficiently to pericentric heterochromatin compared to e-HP1 $\alpha$ ( $52 \%$ vs $31 \%$ of positive cells, respectively, 4 h post-transfection) for comparable levels of expressed proteins along the time course analysis (Fig. 4f). The different efficiencies with which these proteins localized to pericentric heterochromatin support the hypothesis of sumoylation acting as a limiting step to promote HP1 $\alpha$ targeting. In support of this hypothesis, although toxic, the direct fusion of SUMO-1 to HP1 $\alpha$ showed an even more efficient accumulation compared to eHP1 $\alpha$-Ubc9 (Supplementary Fig. 9 and supplemental legend for details). The simplest interpretation is that a higher amount of sumoylated protein provided by HP1 $\alpha$-Ubc9 fusion would allow a rapid targeting to pericentric domains whereas HP1 $\alpha$ alone would be delayed by the time needed to undergo an entire cycle of sumoylation. Overall our data underline the importance of the HP1 $\alpha$ SUMO modification prior to HP1 $\alpha$ targeting to pericentric heterochromatin.

Given that the hinge domain, which shows RNA-binding properties ${ }^{12}$ is the target for sumoylation at multiple sites (Fig. 3b), we generated mutants of e-HP1 $\alpha$ and e-HP1 $\alpha$-Ubc9 lacking the hinge domain and assayed whether they could specifically localize de novo to pericentric heterochromatin in Suv39h dn cells. After transfection of e-HP1 $\alpha \Delta \mathrm{H}$ and e-HP1 $\alpha \Delta \mathrm{H}-\mathrm{Ubc} 9$ constructs in NIH3T3 cells, we found that the hinge domain was perfectly dispensable to localize HP1 $\alpha$ at pre-existing HP1 domains in a 'maintenance assay' (Fig. 5a and b) as reported in Drosophila Kc167 cells ${ }^{29}$. Remarkably, when using Suv39h dn cells 
for our 'de novo' localization assay, after co-transfection with Myc-SUV39H1, the e-HP1 $\alpha$ mutant lacking the hinge domain did not localize at pericentric heterochromatin, in contrast to the wild type protein which did accumulate at these domains (44\% positive cells, Fig. 5c). We verified that all transfected proteins were expressed at comparable levels (Fig. 5d). Even when Ubc9 was fused to the mutant protein, we did not detect localization of e-HP1 $1 \alpha \Delta \mathrm{H}-\mathrm{Ubc} 9$ at pericentric domains. These data clearly show that the hinge domain, the SUMO modification and the association with major RNAs are critical for de novo localization of HP1 $\alpha$ at pericentric heterochromatin.

Based on our data, we propose a model for the de novo targeting and local accumulation of HP1 $\alpha$ at pericentric heterochromatin by a multistep mechanism involving initial SUMO-dependent targeting as a “seeding" step (Fig. 6; "1"). This SUMO modification imposed in the hinge domain of HP1 would leave the CD and CSD available for other interactions with heterochromatin proteins (as represented in Fig 6). Subsequent "chromatin marking" steps would follow, including SUV39-dependent H3K9me3 to ensure HP1 stabilization (Fig. 6; "2") and accumulation through a self-enforcing loop (Fig. 6; “3”)1. Our identification of a specific association between SUMO1-HP1 $\alpha$ and major RNAs in the forward orientation (Fig. 2) provides a molecular basis for the "seeding" step. Such association may help to guide SUMOHP1 $\alpha$ specifically to pericentric heterochromatin. The specificity of the interaction promoted by sumoylation, either by the sequence, by particular structures formed by these RNAs or by know HP1 partners ${ }^{9}$, remains to be determined. Importantly, the HP1-RNA interaction is specific for the forward strand, which is purine rich and may thus adopt a distinct structure. Furthermore, It will be interesting to examine potential SUMO-binding protein candidate ${ }^{30}$. Following the "seeding" event, coordination with SUV39-mediated H3K9 methylation would be key for HP1 $\alpha$ stabilization. While this step could formally be independent of sumoylation, it is possible that SUMO-HP1 and RNA interaction may promote additional interactions with other partners (such as SUV39) and enhance SUV39 enzymatic activity. In this way, the seeding event might favor further stabilization steps to establish a robust system for the maintenance of heterochromatin domains. Given that several proteins involved in heterochromatin stability have been shown to bind SUMO conjugates ${ }^{31}$, it is tempting to speculate that they could also bind SUMOHP1. Future work should address how and where HP1 gets sumoylated and which enzymes trigger both its sumoylation and desumoylation as well as the impact on centromere function. Given the conserved importance of HP1 from fission yeast to mammals ${ }^{3,32}$, further investigation of these issues in various organisms should help to define some general principles.

We detected forward RNAs as long species (several repeat lengths, Fig. 1a), and showed that they localize at the periphery of pericentric domains (Fig. 1b and Supplementary Fig. 2a), however we did not detect small dsRNA corresponding to major satellites, as described for the maintenance of heterochromatin 
in S. pombe $e^{1,33}$. It is intriguing that even in S. pombe primal RNAs have been reported to be important for heterochromatin formation ${ }^{34}$. Although we do not exclude an RNAi pathway in connection with heterochromatin in mammalian cells ${ }^{35,36}$, the fact that we detected long non-coding RNA that are stably located in the nucleus just at the periphery of major satellite domains is very compelling. This is reminiscent of other long nuclear non-coding RNA like the lincRNA HOTAIR, proposed to serve as a modular scaffold for histone modification complexes involved in Polycomb function ${ }^{37}$ or Xist, which is critical to establish the silent state of the inactive $\mathrm{X}$ in mammals. Moreover, Xist RNA is known to be critical in setting up a de novo silent domain at specific times during development ${ }^{38,39}$. In this respect, the situation of early development in mice $e^{40,41}$ is particularly interesting, since a burst of transcription of major RNAs occurs just prior to the formation of HP1 domains of accumulation on the paternal genome $\mathrm{e}^{42}$. Thus, it is tempting to speculate that sumoylation could be critical also at this time and perhaps during other developmental time windows. This mechanism could apply to certain cell types when major rearrangements of the genome occur as observed during spermatogenesis ${ }^{43}$, differentiation ${ }^{44,45}$, reprogramming in the mouse germ line ${ }^{46}$, in specialized cell types like Rod photoreceptor cells ${ }^{47}$, or even in the formation of Senescence Associated Heterochromatin Foci (SAHF) ${ }^{48}$.

Beyond HP1, the concept that a modification like SUMO imposed on a chromatin protein could promote localization guided by a particular transcript in the nucleus, should be explored. One may wonder whether similar mechanisms also apply to the formation of other local domains of accumulation or nuclear compartments such as polycomb or insulator bodies.

\section{ACKNOWLEDGMENTS}

We thank A. Bird, D. Shapiro, R. Hay, T. Jenuwein, R. Losson and J. Seeler for constructs and reagents. We thank W. Faigle for Mass Spectrometry support, G. Cappello for helpful discussions, A. Cook for critical reading and P. Le Baccon at the Curie Imaging Platform. GA's Laboratory is funded by la Ligue Nationale contre le Cancer (Equipe labellisée la Ligue), Curie PIC Programs, the European Network of Excellence Epigenome (LSHG-CT-2004-503433), ACI-2007-Cancéropôle IdF "Breast cancer and Epigenetics", ANR "ECenS" ANR-09-BLAN-0257-01, INCa "GepiG", ERC Advanced Grant 2009-AdG20090506 and DL's Laboratory by le Cancéropôle Ile-de-France and l'INCA.

\section{AUTHOR CONTRIBUTIONS}

C.M., J.-P.Q and G.A. conceived and designed the experiments. C.M., D.B. and J.-P.Q. performed most of the experiments. D.R. and I.V. performed Immuno-DNA FISH and Immuno-RNA FISH. A.V.P. 
performed RNA FISH. F.D., B.L. and D.L. performed and analyzed mass spectrometry data using samples prepared by R.M. and they wrote together the corresponding parts. C.M. generated all figures. C.M., J.P.Q. and G.A. analyzed the data. C.M. and G.A. wrote the paper. All authors contributed to final editing of the manuscript.

\section{COMPETING FINANCIAL INTERESTS}

The authors declare no competing financial interests.

\section{FIGURE LEGENDS}

\section{Figure 1. Strand-specific localization of centromeric RNAs.}

a. Transcription from both strands of major and minor satellite repeats. Top: schematic representation of mouse major and minor satellite repeats and strand specific primers (forward [For], reverse [Rev]) used for analysis by RT-PCR. Bottom: corresponding results for RNAs isolated from NIH3T3 mouse cells. We show PCR reactions in the presence $(+\mathrm{RT})$ or absence $(-\mathrm{RT})$ of reverse transcriptase, or primers, as controls.

b. Nuclear localization of centromeric RNA compared to major and minor satellite DNA in NIH3T3 cells. Left: DNA FISH. Scheme of an acrocentric mouse chromosome with telomeres (black), major (red) and minor (green) satellites. Major (red) and minor (green) satellites are shown along with a merged image of major and minor satellites and DAPI stained DNA. Middle: RNA FISH. We localized major (forward in green, reverse in red) and minor (forward in red, reverse in green) RNAs with strand-specific LNA probes and show a merged image of DAPI and centromeric RNA staining. Insets show magnifications of chromocenters. Right: Immuno-RNA FISH. Forward major RNAs (green) and anti-HP1 $\alpha$ antibodies (red) staining are shown along with a merged image of DAPI and major RNA staining. Scale bar, $10 \mu \mathrm{m}$.

c. HP $1 \alpha$ accumulation at major satellite DNA domains. Immuno-DNA FISH with anti-HP1 $\alpha$ antibodies (green) and major or minor satellite DNA (red) probes. Insets as in b. Scale bar, $10 \mu \mathrm{m}$.

d. RNAs associated with HP1 $\alpha$. ChIP experiments using pre-immune serum (Ctr) or anti-HP1 $\alpha$ antibodies (HP1 $\alpha)$ analysed by strand-specific RT-PCR as indicated. We show PCR reactions with $(+\mathrm{RT})$ or without (-RT) reverse transcriptase, and without cDNA (mock), as controls. The input corresponds to the soluble chromatin prior to ChIP.

Figure 2. SUMO-1-modified HP1 $\alpha$ interacts specifically with forward major RNAs. 
a. Northwestern blot using recombinant GST-HP1 $\gamma$, GST-HP1 $\alpha$ or GST-HP1 $\alpha$ domain fragments and in vitro transcribed radioactively labelled RNAs: forward (F) and reverse (R) major (Maj2) and minor (Min); U1 used as negative control.

b. Experimental scheme.

c. RNA pull down using forward major (Maj2 F) or minor (Min F) RNAs, or no RNA as negative control, in the absence of NEM. We show Western blot analysis with anti-vigilin, anti-RHA, anti-G3bp or antiHP1 $\alpha$ antibodies. Input is $10 \%$ of nuclear extracts.

d. RNA pull down using forward (F) and reverse (R) major (Maj2 F, Maj1 F, Maj2 R) or minor (Min F, Min R) RNAs as baits, or no RNA control, in the presence of NEM. Western blot analysis using antiHP1 $\alpha$, anti-SUMO-1 and anti-SUMO-2/3 antibodies revealed endogenous unmodified HP1 $\alpha$ (HP1 $\alpha$ ), modified-HP1 $\alpha$, SUMO-HP1 $\alpha$ (S-HP1 $\alpha)$ and free SUMO-2/3. Input is $10 \%$ of nuclear extracts. Asterisk $\left(^{*}\right)$ marks SUMO-HP1 $\alpha$ in the input.

\section{Figure 3. Sumoylation of HP1 $\alpha$ occurs at its hinge domain in vitro.}

a. Experimental scheme.

b. HP1 $\alpha$ sumoylation in vitro. Left: Schematic representation of full-length HP1 $\alpha$ and fragments thereof. Right: Western blot analysis of the sumoylation reaction mixture with anti-GST antibodies revealed the positions of SUMO-1-modified full-length HP1 $\alpha$ (S-HP1 $\alpha)$ or fragment domains (S-CD+H and S-H) marked by an asterisk (*).

c. HP1 $\alpha$-Ubc9 sumoylation in vitro. Western blot analysis of the sumoylation reaction mixture with antiGST antibodies revealed the positions of SUMO-1-modified HP1 $\alpha$-Ubc9 (S-HP1 $\alpha$-Ubc9) and unmodified HP1 $\alpha$-Ubc9. Arrow indicates a degradation product of GST-HP1 $\alpha$-Ubc9.

d. Mass spectrometry analysis of the in vitro SUMO-1-modified HP1 $\alpha$ hinge fragment. Shown are the MS (right) and MS/MS (left) fragmentation spectra of the tryptic peptide corresponding to residues 79-97 of SUMO-1 (top right, black) and 83-89 of HP1 $\alpha$ (EKSEGNK; red) where K84 is sumoylated. The precursor ion mass was fragmented and acquired in Qstar (m/z 981.8 [3+]; left) and Orbitrap (m/z 981.77563, mass deviation 2 ppm; right, arrow) mass spectrometers. The majority of the fragment ions could be assigned to the $y$ or $b$ ion series, as annotated in the spectra and peptide sequence (top right).

e. Localization of e-HP1 $\alpha$ and e-HP1 $1 \alpha-U b c 9$ in Triton extracted NIH3T3 cells. Left: Experimental scheme. Right: Immunofluorescence using anti-HA (red) and anti-HP1 $\alpha$ (green) antibodies. Scale bar, 10 $\mu \mathrm{m}$.

Figure 4. Sumoylation of HP1a promotes its targeting and accumulation at pericentric heterochromatin. 
a. Experimental scheme.

b. Endogenous HP1 $\alpha$ (red) and H3K9me3 (green) localization in wild-type and in Suv39h dn cells by immunofluorescence. Transfection of Myc-SUV39H1 in Suv39h dn cells restored HP1 $\alpha$ and H3K9me3 localization in DAPI dense domains. Scale bar, $10 \mu \mathrm{m}$.

c. de novo localization of e-HP1 $\alpha$ or e-HP1 $\alpha$-Ubc9 in Suv39h dn cells by immunofluorescence. Left: HA (red) and DAPI (green) staining with $3 \mathrm{X}$ magnification of selected chromocenters (arrows). Middle: HA (red) and H3K9me3 (green) staining. For each condition, we examined 300 transfected cells and calculated the percentage of cells with HA signal enriched (positive) or not (negative) at pericentric domains. Scale bar, $10 \mu \mathrm{m}$. Right: comparison of protein expression by Western blot revealing HA and $\beta$ actin. Arrow indicates degradation of e-HP1 $\alpha$-Ubc9.

d. Experimental scheme.

e. de novo localization of e-hnRNPC, e-HP1 $\alpha$, e-HP1 $\alpha$-Ubc9C93S and e-HP1 $\alpha$-Ubc9wt in Suv39h dn cells co-transfected with Myc-SUV39H1 by immunofluorescence to reveal HA (red) and Myc (green). The percentage of positive cells was calculated as in c. Scale bar, $10 \mu \mathrm{m}$.

f. Time course analysis of the de novo localization of e-hnRNPC, e-HP1 $\alpha$ and e-HP1 $\alpha-\mathrm{Ubc} 9$ in Suv39h dn cells co-transfected with Myc-SUV39H1. Top: the percentage of positive cells as a function of the time after transfection is represented. Symbols indicate the mean and error bars indicate the standard deviation of three independent experiments (300 co-transfected cells counted in each condition). Bottom: comparison of protein expression as in c. Arrow indicates degradation of e-HP1 $\alpha$-Ubc9.

\section{Figure 5. The hinge domain is required for de novo localization of HP1a at pericentric heterochromatin.}

a. Experimental scheme.

b. Localization of wild type (WT) or mutant $(\Delta \mathrm{H})$ e-HP1 $\alpha$ and e-HP1 $\alpha-U b c 9$ in NIH3T3 cells by immunofluorescence using anti-HA antibodies (red) $24 \mathrm{~h}$ after transfection. Scale bar, $10 \mu \mathrm{m}$.

c. de novo localization of wild type (WT) or mutant $(\Delta \mathrm{H})$ e-HP1 $\alpha$ and e-HP1 $\alpha-\mathrm{Ubc} 9$ in Suv39h dn cells co-transfected with Myc-SUV39H1 by immunofluorescence using anti-HA (red) and anti-Myc (green) antibodies $6 \mathrm{~h}$ after transfection. For each condition, we calculated the percentage of cells with HA signal enriched at pericentric domains (positive cells). Scale bar, $10 \mu \mathrm{m}$.

d. Comparison of protein expression corresponding to experiment in c by Western blot using anti-HA and anti- $\beta$ actin antibodies. Arrows indicate degradation products of e-HP1 $\alpha-\mathrm{Ubc} 9$ and e-HP1 $\alpha \Delta \mathrm{H}-\mathrm{Ubc} 9$.

Figure 6. Model for a de novo HP1 $\alpha$ targeting to pericentric heterochromatin. 
A schematic representation of a nucleus with pericentric domains enriched in HP1 (red) is depicted showing the nuclear non-coding forward major RNA (green) at the periphery. HP1 (red) most likely as part of a complex becomes sumoylated. This SUMO-modified form of HP1 $\alpha$ recognizes and binds to major RNAs (green) at pericentric heterochromatin, providing specificity to the initial targeting of HP1 $\alpha$ to these domains (1). HP1 $\alpha$ stabilization is then ensured by the recognition of H3K9me3 (blue) introduced by SUV39 (light brown) (2). Further HP1 $\alpha$ accumulation involves a "self-enforcing" loop in which new HP1 $\alpha$ directly binds to chromatin by multimerizing with other HP1 $\alpha$ molecules, or by associating with other proteins and/or newly methylated H3K9 (3).

\section{ONLINE METHODS}

Mouse cell lines. We cultured wild type and Suv39h double-null MEFs (provided by T. Jenuwein) ${ }^{27}$ and NIH3T3 cells (ATCC \#CRL-1658) as described ${ }^{2}$. We transfected MEFs and NIH3T3 cells with Nucleofector Kit 2 (Amaxa) and Lipofectamine 2000 (Invitrogen) respectively, according to manufacturer instructions.

Plasmids. We obtained plasmids encoding GFP-SUMO-1 from R. Hay, Myc-SUV39H1 from T. Jenuwein $^{22}$ and GST-HP1 $\alpha$ full-length protein and fragments thereof $(\mathrm{CD}, \mathrm{CD}+\mathrm{H}, \mathrm{H}$ and CSD) from $\mathrm{R}$. Losson $^{49}$. We carried out cloning using standard PCR-based techniques. We made e-HP1 $\alpha$, e-Ubc9 and eSUMO-1 $1 \Delta 6$ constructs by inserting HP1 $\alpha$ (gift of R. Losson), Ubc9 (gift of J. Seeler) and SUMO-1 $\Delta 6$ (from pEGFP-SUMO-1) fused to a HA-tag in C-terminus into pcDNA5 vector (Invitrogen). We generated e-HP1 $\alpha$-Ubc9 and e-HP1 $\alpha$-SUMO-1 constructs by inserting the HP1 $\alpha$ cDNA at the N-terminus of the eUbc9 and the e-SUMO-1 $1 \Delta 6$ plasmids, respectively. To avoid conjugation of e-HP1 $\alpha$-SUMO- 1 into other proteins, we removed the last six C-terminal amino acids of SUMO-1, which contain the diglycine motif required for isopeptide bond formation. We generated HP1 $\alpha$ and e-HP1 $\alpha$-Ubc9C93S point mutants using the QuickChange site-directed mutagenesis kit (Stratagene). To generate e-HP1 $\alpha \Delta \mathrm{H}$ and e-HP1 $\alpha \Delta-\mathrm{Ubc} 9$ mutants, we made truncation contructs of e-HP1 $\alpha$ and e-HP1 $\alpha-\mathrm{Ubc} 9$ in which amino acids M67 to R117 forming the hinge domain were deleted and replaced by a linker of 2 amino acids (ID). These truncation contructs led to the fusion of the chromo domain to the chromoshadow domain. We generated GST-HP1 $\alpha$ hinge-chromoshadow domain and GST-HP1 $\alpha$-Ubc9 by subcloning from GST-HP1 $\alpha$ full-length and eHP1 $\alpha$-Ubc9, respectively. Each mutation and truncation was verified by sequencing.

Antibodies. We used: mouse monoclonal anti-HP1 $\alpha$ (2HP-1H5-AS for immunofluorescence and 2HP2G9-AS for Western blot; 1:1,000), anti-HP1 $\beta$ (1MOD-1A9-AS; 1:1,000), and anti-HP1 $\gamma$ (2MOD-1G6- 
AS; 1:1,000) all from Euromedex, rabbit polyclonal anti-vigilin (from D. Shapiro ${ }^{50} ; 1: 3,000$ ), rabbit polyclonal anti-RHA (PA-001, Vaxron; 1:10,000), mouse monoclonal anti-SUMO-1 (\#33-2400; 1:500) and rabbit polyclonal anti-SUMO-2/3 (\#51-9100; 1:250) both from Zymed, rat monoclonal anti-HA (\#1867423, Roche; 1:2,000 for Western blot and 1:250 for immunofluorescence), mouse monoclonal antiG3bp (\#611126, BD Biosciences; 1:1,000), mouse monoclonal anti-Myc (ab32, Abcam; 1:500), rabbit polyclonal anti-GST (ab9085 Abcam; 1:1,000), rabbit polyclonal anti-GFP (sc-8334, Santa-Cruz; 1:500), rabbit polyclonal anti-H3K9me3 (\#07-442, Upstate; 1:500), mouse monoclonal anti- $\beta$ actin (\#A5441 Sigma; 1:20,000). For chromatin immunoprecipitations we used a rabbit polyclonal anti-HP1 $\alpha$ antibody generated against the full length GST-HP1 $\alpha$ protein (Agro-Bio).

Nuclear extracts. After incubation in hypotonic buffer A (20 mM Hepes-KOH pH 7.8, $5 \mathrm{mM}$ potassium acetate, $0.5 \mathrm{mM} \mathrm{MgCl} 2,0.5 \mathrm{mM}$ DTT) for $10 \mathrm{~min}$ at $4{ }^{\circ} \mathrm{C}$, we disrupted NIH3T3 cells by 25 strokes with a dounce homogenizer and separated nuclei from the soluble proteins by centrifugation at $1600 \mathrm{~g}$. After incubation of nuclear pellets in buffer A containing $615 \mathrm{mM} \mathrm{NaCl}$ for $1.5 \mathrm{~h}$ at $4^{\circ} \mathrm{C}$, followed by centrifugation at $14000 \mathrm{~g}$ for $20 \mathrm{~min}$, we collected supernatant as the nuclear extract that we aliquoted and flash froze in liquid nitrogen. All buffers contained protease and phosphatase inhibitors $(10 \mu \mathrm{g} / \mathrm{ml}$ pepstatin, $10 \mu \mathrm{g} / \mathrm{ml}$ leupeptin, $100 \mu \mathrm{M}$ PMSF, $5 \mathrm{mM}$ sodium fluoride, $10 \mathrm{mM} \beta$-glycerophosphate, plus or minus $20 \mathrm{mM}$ N-ethylmaleimide [NEM; Sigma] when indicated).

Centromeric RNA pull down. The Maj9-2 and Min5-1 pCR4 plasmids contain 542-bp of the mouse major satellite DNA and 162-bp of minor satellite DNA, respectively (provided by T. Jenuwein) ${ }^{51}$. We subcloned a cDNA encoding a 234-bp repeat unit of the mouse major satellite DNA from pUC19-Sat15 (provided by A. Bird) into the pBS vector (Stratagene). We obtained biotinylated major (Maj1 from Sat15-pBS and Maj2 from Maj9-2 pCR4) and minor (Min from Min5-1 pCR4) RNAs by in vitro transcription with T7 or T3 RNA polymerases (Promega) in the presence of biotin RNA labelling mix (Roche), at $37^{\circ} \mathrm{C}$ for $2 \mathrm{~h}$. After DNA digestion by RNase-free DNase I (Promega), we removed unincorporated rNTPs by a Sephadex G-50 quick spin column (Roche). For RNA pull down, we incubated 2 $\mu \mathrm{g}$ of biotinylated RNA with nuclear extracts in binding buffer $(20 \mathrm{mM}$ Hepes $\mathrm{pH} 7.6,100 \mathrm{mM} \mathrm{KCl}, 2$ mM EDTA, 0.01\% Nonidet P-40, 1\% gelatin) with $200 \mu \mathrm{g} / \mathrm{ml}$ tRNA, $4 \mathrm{mg} / \mathrm{ml}$ heparin, $80 \mathrm{U}$ RNasin, plus or minus $20 \mathrm{mM} \mathrm{NEM}$ as indicated, for $30 \mathrm{~min}$ at room temperature. For each binding reaction we used $100 \mu \mathrm{l}$ of streptavidin-coated magnetic beads (Dynabeads; Invitrogen) for $1 \mathrm{~h}$ at room temperature, on a rotating wheel. After 4 washes with binding buffer containing $20 \mu \mathrm{g} / \mathrm{ml}$ tRNA and $0.1 \%$ Tween-20, followed by one more wash with $20 \mathrm{mM}$ Hepes pH 7.6, plus $50 \mathrm{mM} \mathrm{KCl}$, we eluted bound proteins with 
SDS-PAGE loading buffer and ran samples on NuPAGE 4-12\% Bis-Tris gels (Invitrogen) with MOPS running buffer (Invitrogen). We stained gels with Coomassie brilliant blue.

Immunoprecipitations and Western blotting. We lysed NIH3T3 cells $48 \mathrm{~h}$ post-transfection, with lysis buffer (50 mM Tris-HCl pH 7.5, $150 \mathrm{mM} \mathrm{NaCl}, 5 \mathrm{mM}$ EDTA, $15 \mathrm{mM} \mathrm{MgCl}, 1 \%$ Nonidet P-40, and $0.75 \%$ sodium deoxycholate) supplemented with protease and phosphatase inhibitors, and $20 \mathrm{mM}$ NEM (Sigma). We incubated cell lysates corresponding to $4 \times 10^{6}$ cells with $40 \mu \mathrm{l}$ of monoclonal anti-HA agarose-conjugated beads (Roche) for $2 \mathrm{~h}$ at $4^{\circ} \mathrm{C}$. After washing the beads with lysis buffer, we eluted the immunocomplexes with SDS-PAGE loading buffer, and resolved proteins by $4-12 \%$ Bis-Tris NuPAGE gels (Invitrogen), and transferred to nitrocellulose membranes (Protran). For visualization of proteins after Western blots with the indicated antibodies we used the Super Signal detection kit (Pierce).

Northwestern blotting. We resolved recombinant full-length GST-HP1 $\gamma$ and GST-HP1 $\alpha$ full-length and fragments thereof by SDS-PAGE and transferred them to nitrocellulose. We incubated the membranes with in vitro transcribed radioactively labelled RNAs in buffer containing $20 \mathrm{mM}$ Hepes pH 7.6, $100 \mathrm{mM}$ $\mathrm{KCl}, 2 \mathrm{mM}$ EDTA, $0.01 \%$ Nonidet P-40, overnight at $23^{\circ} \mathrm{C}$. After 3 washes with the same buffer, we visualized bound radioactively labelled RNAs by autoradiography.

Immunofluorescence microscopy. We processed cells for immunostaining as described ${ }^{11}$. We used an epifluorescence photo-microscope (DM6000B, Leica) piloted with Metamorph software, an x63 (NA 1.32) objective lens and an HQ2 CoolSnap camera (Photometrics) for image acquisition. For all time course studies, we performed three independent experiments. For spot formation analysis, after image acquisition, we draw a scan line across the nucleus and measured the relative intensity of fluorescence across this line for signals corresponding to e-HP1 $\alpha$ and Myc-SUV39H1 (ImageJ software). For ImmunoRNA FISH, we acquired 50 optical sections separated by $0.2 \mu \mathrm{m}$ with an Imager.Z1 microscope (Zeiss) piloted with Metamorph software and made $\mathrm{Z}$ projections. We quantified the localization of forward major transcripts at the periphery of pericentric heterochromatin domains from 3D image series (z-step $0.2 \mu \mathrm{m}$ ) of major RNA FISH/DAPI staining acquired on a Delta Vision system (Applied Precision, 100x objective). For each nucleus, we manually scored the total number of RNA foci $\left(f_{\text {tot }}\right)$ and the number of RNA foci at the periphery of DAPI dense pericentric domains $\left(f_{\text {per }}\right)$ using ImageJ software and the Image $5 \mathrm{D}$ plugin to allow co-visualization in 3D of the RNA FISH and DAPI signals. We determined the volume of the nuclei $\left(\mathrm{V}_{\mathrm{nuc}}\right)$ and the volume of the individual pericentric domains ( $\mathrm{V}_{\text {dom, }}$, DAPI dense) using the ImageJ software and the 3D Object Counter plugin. We calculated the volume of peripheral pericentric domain $\left(\mathrm{V}_{\text {per }}\right)$ as the difference between the volume corresponding to the pericentric domain with an 
increase of $20 \%$ of the radius of the domain ( $\mathrm{V}_{\mathrm{dom} 20 \%}$, assuming a spherical form) and the volume of the domain $\left(\mathrm{V}_{\text {per }}=\mathrm{V}_{\text {dom } 20 \%}-\mathrm{V}_{\text {dom }}\right)$. We obtained the concentration of RNA foci (foci/ $\left.\mu \mathrm{m}^{3}\right)$ at the periphery from the ratio $f_{\text {per }} / V_{\text {per }}$ and in the nucleus from the ratio $f_{\text {tot }} /\left(V_{\text {nuc }}-V_{\text {dom }}\right)$. We removed the volume of the pericentric domains from total volume given that RNA foci are never found within domains.

RNA and DNA FISH. For RNA FISH, after extraction with $0.5 \%$ Triton X-100 in CSK buffer (10 mM Pipes pH7, $100 \mathrm{mM} \mathrm{NaCl}, 300 \mathrm{mM}$ sucrose, $3 \mathrm{mM} \mathrm{MgCl}$, supplemented with $10 \mathrm{mM}$ Vanadyl ribonucleoside complex (VRC)) for 5min on ice, we fixed cells in 3\% paraformaldehyde in PBS for 12 min and stored them in $70 \% \mathrm{EtOH}$ at $-20^{\circ} \mathrm{C}$ overnight. Following dehydration in $80 \%, 95 \%$ and $100 \%$ EtOH, we carried out hybridization with $0.4 \mu \mathrm{M}$ locked nucleic acid (LNA) fluorescent probes (Exiqon) in 50\% formamide (Sigma), 2x SSC (Sigma), 10\% dextran sulfate (Fluka), $10 \mathrm{mM}$ VRC (NEB) and 2 $\mathrm{mg} / \mathrm{mL}$ BSA (NEB) in a humid chamber for $35 \mathrm{~min}$ at $37^{\circ} \mathrm{C}$. After 3 washes in $0.1 \mathrm{x} \mathrm{SSC}$ for $5 \mathrm{~min}$ at $60^{\circ} \mathrm{C}$, we stained DNA with DAPI staining and mounted the cells in Vectashield (Vector Laboratories). For Immuno-RNA FISH, we performed RNA FISH as described above. After post-hybridization washes, we post-fixed cells in 3\% paraformaldehyde in PBS for $12 \mathrm{~min}$ and processed them for immunostaining. DNA FISH and Immuno-DNA FISH were performed as described ${ }^{2}$, except that the hybridization mix contained LNA fluorescent probes $(0.1 \mu \mathrm{M})$ and we performed post-hybridization washes in $0.1 \mathrm{X}$ SSC (3 times for $5 \mathrm{~min}$ ) at $60^{\circ} \mathrm{C}$. The sequences of the LNA fluorescent probes are listed in Supplementary Table 2.

RT-PCR analysis. We performed RT-PCR analysis on HP1 $\alpha$-associated RNAs and on total RNA extracted from $3 \mathrm{~T} 3$ cells with Trizol (Invitrogen). We digested genomic DNA by incubation with DNase 1 (Sigma). We synthesized first strand cDNA from $1 \mu \mathrm{g}$ RNA in $20 \mu \mathrm{l}$ buffer containing $1 \mu \mathrm{M}$ forward (For) or reverse (Rev) specific primers for major or minor satellites, $0.5 \mathrm{mM}$ dNTPs, $40 \mathrm{U} / \mu 1 \mathrm{RNasin}$ and $10 \mathrm{U} / \mu 1$ of SuperScript II reverse transcriptase (Qiagen). We then amplified the generated cDNA by PCR using 1/2000 and 1/250 dilutions of major and minor cDNA respectively, and a PCR Master Kit (Roche) supplemented with $0.5 \mu \mathrm{M}$ specific primers during 45 cycles. Primer sequences are listed in Supplementary Table 2 .

Chromatin Immunoprecipitation (ChIP). We extracted NIH3T3 cells with Triton X-100 to remove soluble proteins as previously described ${ }^{52}$ and cross-linked them with $1.5 \%$ formaldehyde in PBS for 20 min at room temperature. We then added $125 \mathrm{mM}$ glycine for $20 \mathrm{~min}$ to quench any residual formaldehyde. After one wash with PBS, we collected the cells by scraping and resuspended them in ChIP buffer (20 mM Tris- $\mathrm{HCl}$ at pH 7.5, $200 \mathrm{mM} \mathrm{NaCl}, 0.2 \%$ Nonidet P-40, 1\% gelatin and $80 \mathrm{U}$ RNasin). We 
then sonicated the cells seven times for 30 seconds on high intensity (Bioruptor sonicator, CosmoBio Inc.) and centrifuged at $10000 \mathrm{~g}$ for $5 \mathrm{~min}$. We incubated approximately 200-300 $\mu \mathrm{g}$ of supernatant containing the soluble chromatin with $20 \mu \mathrm{l}$ of sera against HP1 $\alpha$ raised in the laboratory for $3 \mathrm{~h}$ at $4^{\circ} \mathrm{C}$. We used preimmune sera as negative control. Then, we added $100 \mu$ of Protein-A sepharose slurry (50\% W/V, Amersham Biosciences) and incubated for $3 \mathrm{~h}$ at $4^{\circ} \mathrm{C}$ on a rotating wheel. We recovered the immunoprecipitated chromatin by centrifugation, washed it five times with $1 \mathrm{ml}$ of ChIP buffer and resuspended it in $10 \mathrm{mM}$ Tris- $\mathrm{HCl}$ at $\mathrm{pH} 7.5,1 \mathrm{mM}$ EDTA, $0.2 \%$ SDS. We reversed the crosslink by incubation at $65^{\circ} \mathrm{C}$ overnight. We then extracted the HP1 $\alpha$-associated RNAs with Trizol (Invitrogen) and we performed RT-PCR analysis.

In vitro sumoylation assays. We produced recombinant proteins in E. coli by expressing constructs corresponding to the GST-HP1 $\alpha$ full-length protein and fragments thereof and GST-HP1 $\alpha-\mathrm{Ubc} 9$. We utilized the recombinant proteins in in vitro sumoylation reactions, using the SUMOlink kits (Active Motif), using manufacturer instructions. We validated sumoylation of HP1 $\alpha$ and HP1 $1 \alpha$-Ubc9 by Western blot with anti-GST antibodies. For analysis of the sumoylated GST-HP1 $\alpha$ hinge fragment by mass spectrometry, we used a total of $5 \mu \mathrm{g}$ of GST-HP1 $\alpha$ hinge and $10 \mu \mathrm{g}$ of SUMO-1 protein. We ran $90 \%$ of the reaction mixture on a 4-12\% Bis-Tris NuPAGE gel (Invitrogen) and stained the gel overnight with LabSafe GEL Blue stain (Biosciences) for later mass spectrometry analysis. We used the remaining 10\% of the sumoylation mixture for Western blotting with rabbit anti-GST and rabbit anti-SUMO-1 (Active Motif; 1:4,000 dilution) antibodies.

Mass Spectrometry. We reduced, alkylated and trypsin digested slices (1 mm wide) cut out from Coomassie blue-stained gels as previously described ${ }^{53}$. We dried extracted peptides, resolubilized them in solvent A (95/5 water/acetonitrile, $0.1 \%$ formic acid) prior to liquid chromatography tandem mass spectrometry (LC-MS/MS) analysis. We concentrated and separated them on a LC-Packings system (Dionex S.A.) coupled to the nano-electrospray II ionization interface of a QSTAR Pulsar i (Applied Biosystems/MDS Sciex). HPLC mobile phases contained solvent A and solvent B [20/80: water/acetonitrile, $0.085 \%$ formic acid]. We eluted bound peptides with a gradient of $5-50 \%$ of solvent $\mathrm{B}$. We used information-dependent acquisition (IDA) to acquire MS/MS data, with experiments designed such that the two most abundant peptides were subject to collision-induced dissociation (CID). We analyzed twice the data from the IDA experiments by using MASCOT software (Matrix Science, London) on an internal server, first without taxonomic restriction to reveal the presence of proteins of interest and mammalian contaminants, then again the "Mus musculus" (mouse) database of the National Center for Biotechnology Information nr (National Library of Medicine, Bethesda, 200505 02, 2452561 and 41362 
protein entries). We converted the RAW files to the Mascot Generic Format (MGF) and submitted them to the Mascot search engine (version 1.0). We used the following parameters in the database search: Full trypsin enzyme specificity; Missed cleavages allowed $=1$, Peptide mass tolerance $=0.8 \mathrm{Da}$; Fragment ion tolerance $=1 \mathrm{Da}$; Monoisotopic molecular weight for both peptide and fragment ion masses, b/y ion search, and fixed carbamidomethyl cysteine modification. We manually validated all data using myProMS ${ }^{54}$.

For SUMO-1 LC-MS/MS experiments, we used two different MS platforms in parallel. We achieved peptide concentration and separation using an actively split capillary HPLC system (Ultimate 3000, Dionex, Germering, Germany) connected to each MS platform. The first platform was a quadrupole timeof-flight (Q-TOF) mass spectrometer (QSTAR Elite, Applied Biosystems/MDS Sciex). We acquired a TOF-MS survey scan for $1 \mathrm{sec}$ over a mass range of 800-1200 m/z. We used an IDA method to acquire product ion scans on the three most intense 3+ ions per cycle over a mass range of 65-2000 m/z, excluding previously gated ions for $60 \mathrm{sec}$. We used a Smart setting of 2.0. The second platform was an LTQOrbitrap XL mass spectrometer (Thermo Fisher Scientific, Bremen, Germany) equipped with a nanospray source using a Pico-Tip (10 $\mu \mathrm{m}$ i.d., New Objectives). We set the spray voltage to $2.2 \mathrm{kV}$ and the temperature of the heated capillary to $200^{\circ} \mathrm{C}$. The mass spectrometer was operated in data-dependent mode to automatically acquire MS and MS/MS spectra. We acquired full scan survey spectra $(\mathrm{m} / \mathrm{z}$ 615-1200) in the Orbitrap with a resolution of 100,000 at $\mathrm{m} / \mathrm{z} 400$ after accumulation of 1,000,000 charges. We sequentially isolated the five most intense ions and fragmented them in the linear ion trap by collisioninduced dissociation after accumulation of 30,000 ions (normalized collision energy 35\%). Maximum inject times were $500 \mathrm{msec}$ for full scans and $200 \mathrm{msec}$ for MS/MS scans. Dynamic exclusion was enabled with exclusion duration of $120 \mathrm{sec}$. We calculated and used the monoisotopic m/z values for SUMO-1GST-HP1 $\alpha$ hinge branched precursor peptides as described ${ }^{55}$ to search for the corresponding ions (assignment was confirmed by manually interpreting all MS/MS spectra). We manually validated all reported MS/MS spectra. We considered only branched peptides having an extensive coverage of $\mathrm{b}$ and/or $\mathrm{y}$ ions. It was assumed that modified (sumoylated) lysines cannot be cleaved by trypsin, and one trypsin missed cleavage was allowed.

\section{REFERENCES}

1. Grewal, S.I. \& Jia, S. Heterochromatin revisited. Nat Rev Genet 8, 35-46 (2007).

2. Guenatri, M., Bailly, D., Maison, C. \& Almouzni, G. Mouse centric and pericentric satellite repeats form distinct functional heterochromatin. J Cell Biol 166, 493-505 (2004).

3. Probst, A.V., Dunleavy, E. \& Almouzni, G. Epigenetic inheritance during the cell cycle. Nat Rev Mol Cell Biol 10, 192-206 (2009). 
4. Bannister, A.J. et al. Selective recognition of methylated lysine 9 on histone H3 by the HP1 chromo domain. Nature 410, 120-4 (2001).

5. Lachner, M., O'Carroll, D., Rea, S., Mechtler, K. \& Jenuwein, T. Methylation of histone H3 lysine 9 creates a binding site for HP1 proteins. Nature 410, 116-20 (2001).

6. Strahl, B.D. \& Allis, C.D. The language of covalent histone modifications. Nature 403, 41-5 (2000).

7. Jenuwein, T. \& Allis, C.D. Translating the histone code. Science 293, 1074-80 (2001).

8. Peng, H., Ivanov, A.V., Oh, H.J., Lau, Y.F. \& Rauscher, F.J., 3rd. Epigenetic gene silencing by the SRY protein is mediated by a KRAB-O protein which recruits the KAP1 co-repressor machinery. J Biol Chem (2009).

9. Quivy, J.P. et al. A CAF-1 dependent pool of HP1 during heterochromatin duplication. EMBO J 23, 3516-26 (2004).

10. Lomberk, G., Bensi, D., Fernandez-Zapico, M.E. \& Urrutia, R. Evidence for the existence of an HP1-mediated subcode within the histone code. Nat Cell Biol 8, 407-15 (2006).

11. Maison, C. et al. Higher-order structure in pericentric heterochromatin involves a distinct pattern of histone modification and an RNA component. Nat Genet 30, 329-34 (2002).

12. Muchardt, C. et al. Coordinated methyl and RNA binding is required for heterochromatin localization of mammalian HP1alpha. EMBO Rep 3, 975-81 (2002).

13. Rudert, F., Bronner, S., Garnier, J.M. \& Dolle, P. Transcripts from opposite strands of gamma satellite DNA are differentially expressed during mouse development. Mamm Genome 6, 76-83 (1995).

14. Lu, J. \& Gilbert, D.M. Proliferation-dependent and cell cycle regulated transcription of mouse pericentric heterochromatin. J Cell Biol 179, 411-21 (2007).

15. Kalitsis, P. \& Choo, K.H.A. Centromere DNA of higher eukaryotes. in The Centromere (ed. Choo, K.H.A.) 97-140 (Oxford University Press, New York, 1997).

16. Heard, E. \& Bickmore, W. The ins and outs of gene regulation and chromosome territory organisation. Curr Opin Cell Biol 19, 311-6 (2007).

17. Deng, Z., Norseen, J., Wiedmer, A., Riethman, H. \& Lieberman, P.M. TERRA RNA binding to TRF2 facilitates heterochromatin formation and ORC recruitment at telomeres. Mol Cell 35, 40313 (2009).

18. Wang, Q., Zhang, Z., Blackwell, K. \& Carmichael, G.G. Vigilins bind to promiscuously A-to-Iedited RNAs and are involved in the formation of heterochromatin. Curr Biol 15, 384-91 (2005).

19. Kuroda, M.I., Kernan, M.J., Kreber, R., Ganetzky, B. \& Baker, B.S. The maleless protein associates with the $\mathrm{X}$ chromosome to regulate dosage compensation in Drosophila. Cell 66, 93547. (1991).

20. Irvine, K., Stirling, R., Hume, D. \& Kennedy, D. Rasputin, more promiscuous than ever: a review of G3BP. Int J Dev Biol 48, 1065-77 (2004).

21. Shin, J.A. et al. SUMO modification is involved in the maintenance of heterochromatin stability in fission yeast. Mol Cell 19, 817-28 (2005).

22. Aagaard, L. et al. Functional mammalian homologues of the Drosophila PEV- modifier Su(Var)39 encode centromere-associated proteins which complex with the heterochromatin component M31. EMBO J 18, 1923-1938 (1999).

23. Hay, R.T. SUMO: a history of modification. Mol Cell 18, 1-12 (2005).

24. Meulmeester, E. \& Melchior, F. Cell biology: SUMO. Nature 452, 709-11 (2008).

25. Park-Sarge, O.K. \& Sarge, K.D. Detection of sumoylated proteins. Methods Mol Biol 464, 255-65 (2009).

26. Jakobs, A. et al. Ubc9 fusion-directed SUMOylation (UFDS): a method to analyze function of protein SUMOylation. Nat Methods 4, 245-50 (2007).

27. Peters, A. et al. Loss of the Suv39h histone methyltransferases impairs mammalian heterochromatin and genome stability. Cell 107, 323-337 (2001). 
28. Loyola, A., Bonaldi, T., Roche, D., Imhof, A. \& Almouzni, G. PTMs on H3 variants before chromatin assembly potentiate their final epigenetic state. Mol Cell 24, 309-16 (2006).

29. Smothers, J.F. \& Henikoff, S. The hinge and chromo shadow domain impart distinct targeting of HP1-like proteins. Mol Cell Biol 21, 2555-69 (2001).

30. Kerscher, O. SUMO junction-what's your function? New insights through SUMO-interacting motifs. EMBO Rep 8, 550-5 (2007).

31. Ouyang, J., Shi, Y., Valin, A., Xuan, Y. \& Gill, G. Direct binding of CoREST1 to SUMO-2/3 contributes to gene-specific repression by the LSD1/CoREST1/HDAC complex. Mol Cell 34, 145-54 (2009).

32. Allshire, R.C. \& Karpen, G.H. Epigenetic regulation of centromeric chromatin: old dogs, new tricks? Nat Rev Genet 9, 923-37 (2008).

33. Moazed, D. Small RNAs in transcriptional gene silencing and genome defence. Nature 457, 41320 (2009).

34. Blankenship, J.T. \& Wieschaus, E. Two new roles for the Drosophila AP patterning system in early morphogenesis. Development 128, 5129-38. (2001).

35. Kanellopoulou, C. et al. Dicer-deficient mouse embryonic stem cells are defective in differentiation and centromeric silencing. Genes Dev 19, 489-501 (2005).

36. Murchison, E.P., Partridge, J.F., Tam, O.H., Cheloufi, S. \& Hannon, G.J. Characterization of Dicer-deficient murine embryonic stem cells. Proc Natl Acad Sci U S A 102, 12135-40 (2005).

37. Tsai, M.C. et al. Long Noncoding RNA as Modular Scaffold of Histone Modification Complexes. Science (2010).

38. Savarese, F., Flahndorfer, K., Jaenisch, R., Busslinger, M. \& Wutz, A. Hematopoietic precursor cells transiently reestablish permissiveness for X inactivation. Mol Cell Biol 26, 7167-77 (2006).

39. Agrelo, R. et al. SATB1 defines the developmental context for gene silencing by Xist in lymphoma and embryonic cells. Dev Cell 16, 507-16 (2009).

40. Santos, F., Peters, A.H., Otte, A.P., Reik, W. \& Dean, W. Dynamic chromatin modifications characterise the first cell cycle in mouse embryos. Dev Biol 280, 225-36 (2005).

41. Puschendorf, M. et al. PRC1 and Suv39h specify parental asymmetry at constitutive heterochromatin in early mouse embryos. Nat Genet 40, 411-20 (2008).

42. Probst, A.V. et al. A strand-specific burst in transcription of pericentric satellites is required for chromocenter formation and early mouse development. Dev Cell 19, 625-38 (2010).

43. Haaf, T. \& Ward, D.C. Higher order nuclear structure in mammalian sperm revealed by in situ hybridization and extended chromatin fibers. Exp Cell Res 219, 604-11 (1995).

44. Mayer, R. et al. Common themes and cell type specific variations of higher order chromatin arrangements in the mouse. BMC Cell Biol 6, 44 (2005).

45. Terranova, R., Sauer, S., Merkenschlager, M. \& Fisher, A.G. The reorganisation of constitutive heterochromatin in differentiating muscle requires HDAC activity. Exp Cell Res 310, 344-56 (2005).

46. Hajkova, P. et al. Chromatin dynamics during epigenetic reprogramming in the mouse germ line. Nature 452, 877-81 (2008).

47. Solovei, I. et al. Nuclear architecture of rod photoreceptor cells adapts to vision in mammalian evolution. Cell 137, 356-68 (2009).

48. Narita, M. et al. Rb-mediated heterochromatin formation and silencing of E2F target genes during cellular senescence. Cell 113, 703-16 (2003).

49. Nielsen, A.L. et al. Heterochromatin formation in mammalian cells: interaction between histones and HP1 proteins. Mol Cell 7, 729-39 (2001).

50. Dodson, R.E. \& Shapiro, D.J. Vigilin, a ubiquitous protein with $14 \mathrm{~K}$ homology domains, is the estrogen-inducible vitellogenin mRNA 3'-untranslated region-binding protein. J Biol Chem 272, 12249-52 (1997).

51. Lehnertz, B. et al. Suv39h-mediated histone H3 lysine 9 methylation directs DNA methylation to major satellite repeats at pericentric heterochromatin. Curr Biol 13, 1192-200 (2003). 
52. Martini, E., Roche, D.M., Marheineke, K., Verreault, A. \& Almouzni, G. Recruitment of phosphorylated chromatin assembly factor 1 to chromatin after UV irradiation of human cells. $J$ Cell Biol 143, 563-75 (1998).

53. Fevrier, B. et al. Cells release prions in association with exosomes. Proc Natl Acad Sci U S A 101, 9683-8 (2004).

54. Poullet, P., Carpentier, S. \& Barillot, E. myProMS, a web server for management and validation of mass spectrometry-based proteomic data. Proteomics 7, 2553-6 (2007).

55. Matic, I. et al. In vivo identification of human small ubiquitin-like modifier polymerization sites by high accuracy mass spectrometry and an in vitro to in vivo strategy. Mol Cell Proteomics 7, 132-44 (2008). 

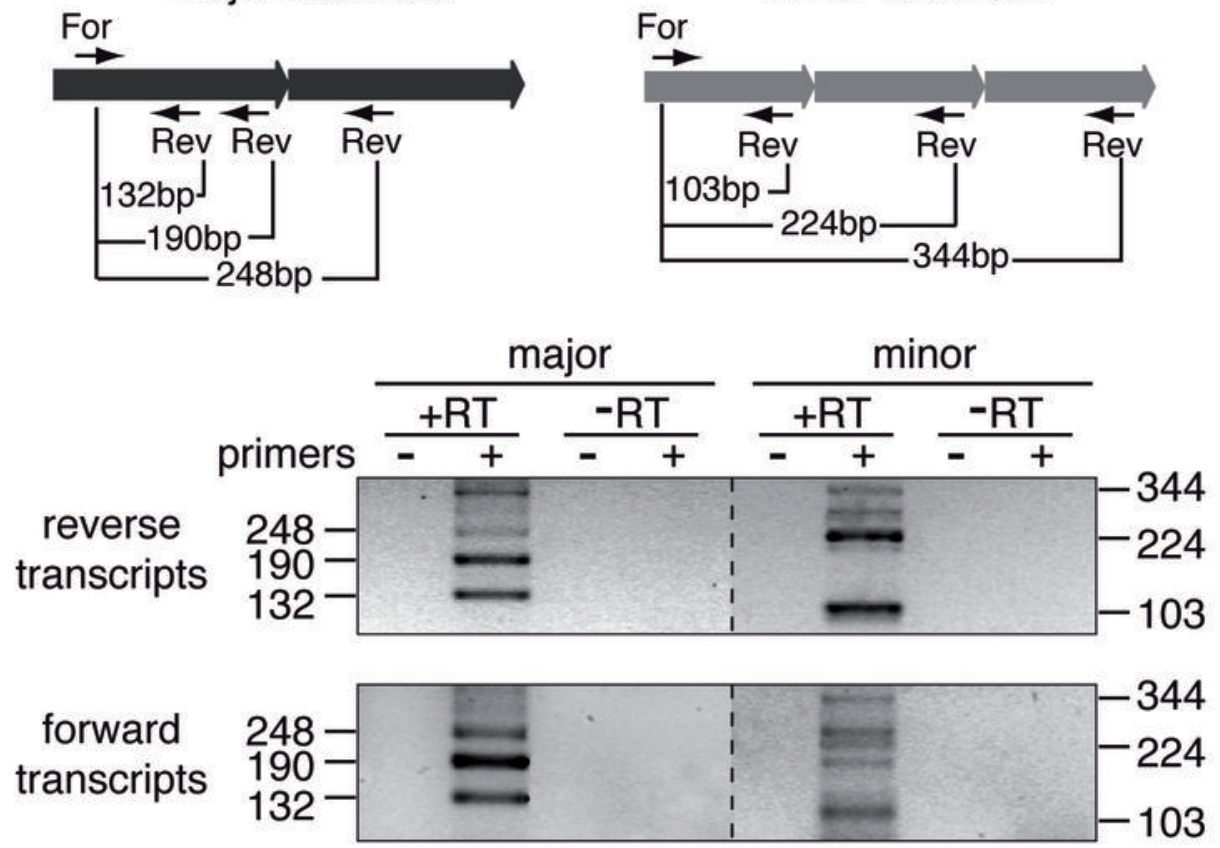

DNA FISH
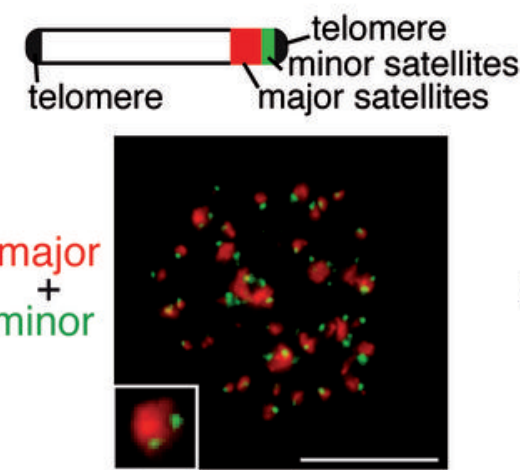

$\mathrm{m}$
$\mathrm{m}$
$\mathrm{D}$

major $+$ mino and DAPI
RNA FISH

major RNA forward + reverse

RNA

RNA and

DAPI minor RNA forward + reverse

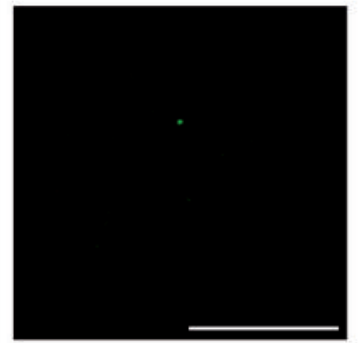

Immuno-DNA FISH
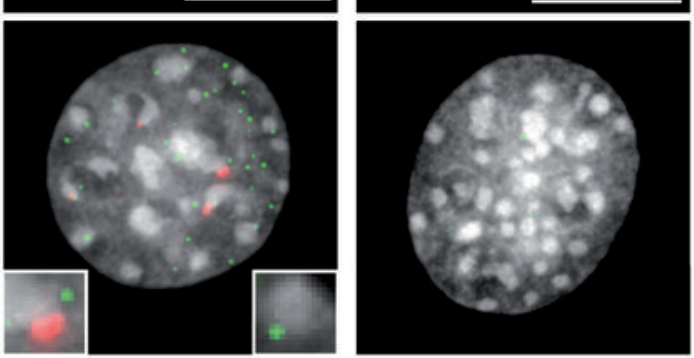

Immuno-RNA FISH

major RNA

forward

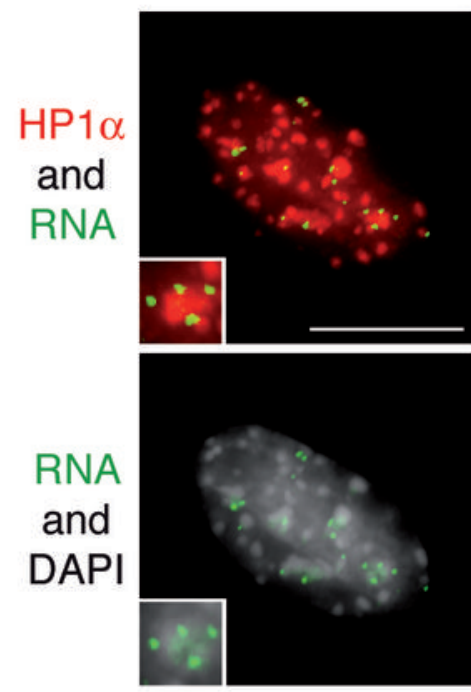

HP1 $\alpha$ and DNA minor DNA

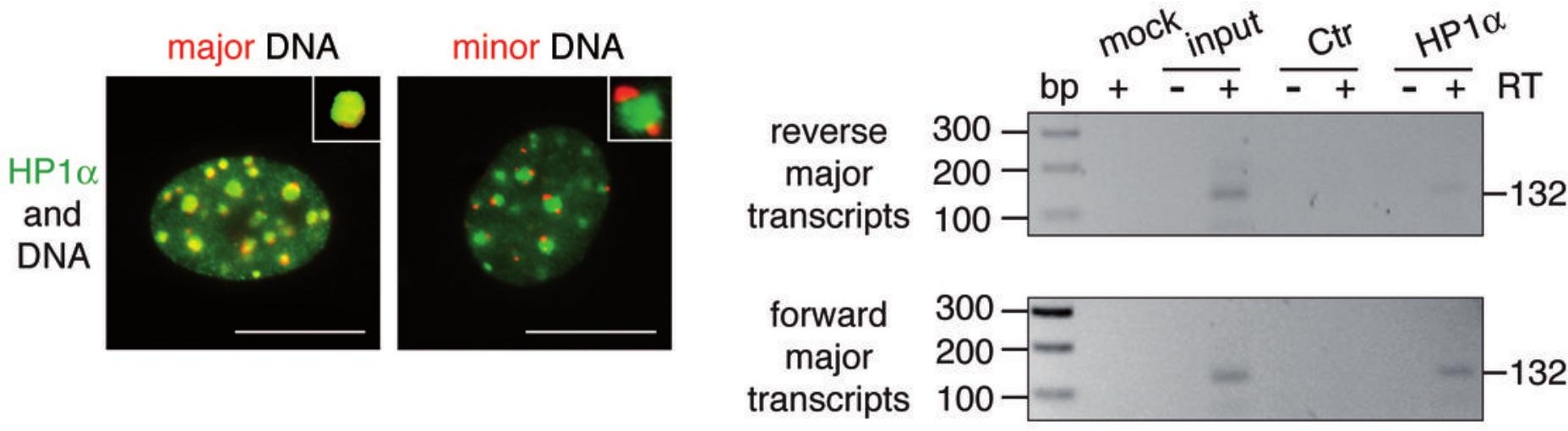

d

HP1 $\alpha$ ChIP

Maison et al. Figure 1 
$\cdot$ e-HP1 $\alpha$

- e-HP1 $\alpha \Delta \mathrm{H}$

-e-HP1 $\alpha$-Ubc9

-e-HP1 $\alpha \Delta \mathrm{H}-U b c 9$

b

\begin{tabular}{|l|c|c|}
\hline $\mathrm{CD}$ & $\mathrm{H}$ & $\mathrm{CSD}$ \\
\hline $\mathrm{CD}$ & $\mathrm{CSD}$ & \\
\hline $\mathrm{CD}$ & $\mathrm{H}$ & $\mathrm{CSD}-U b c 9$ \\
\hline $\mathrm{CD}$ & \multicolumn{1}{|c|}{ CSD } & $-\mathrm{Ubc} 9$ \\
\hline
\end{tabular}

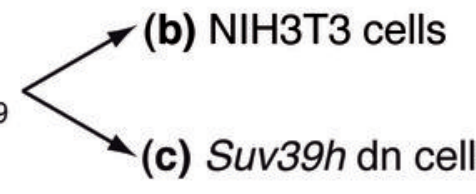

$\rightarrow$ Transfection

Co-transfection

NIH3T3 cells
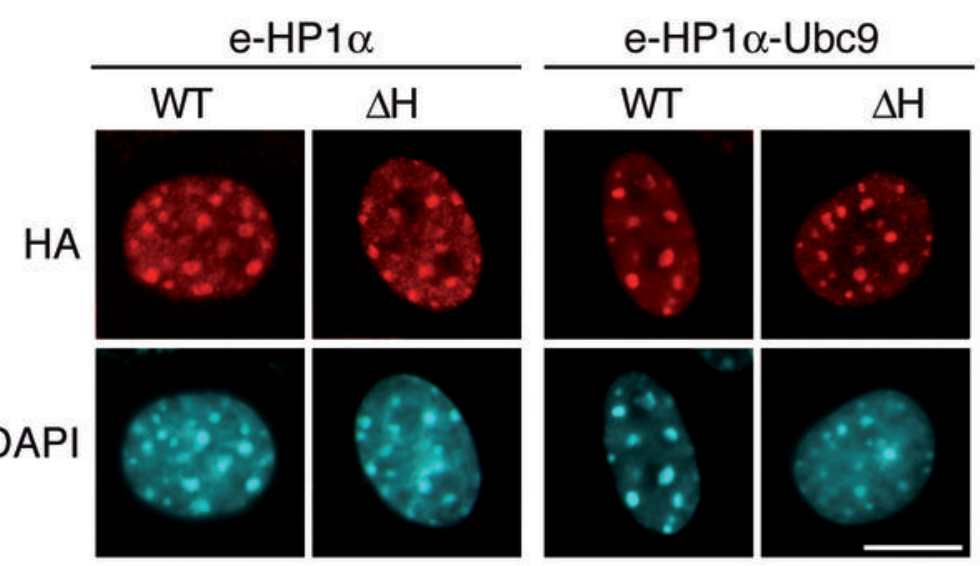

d

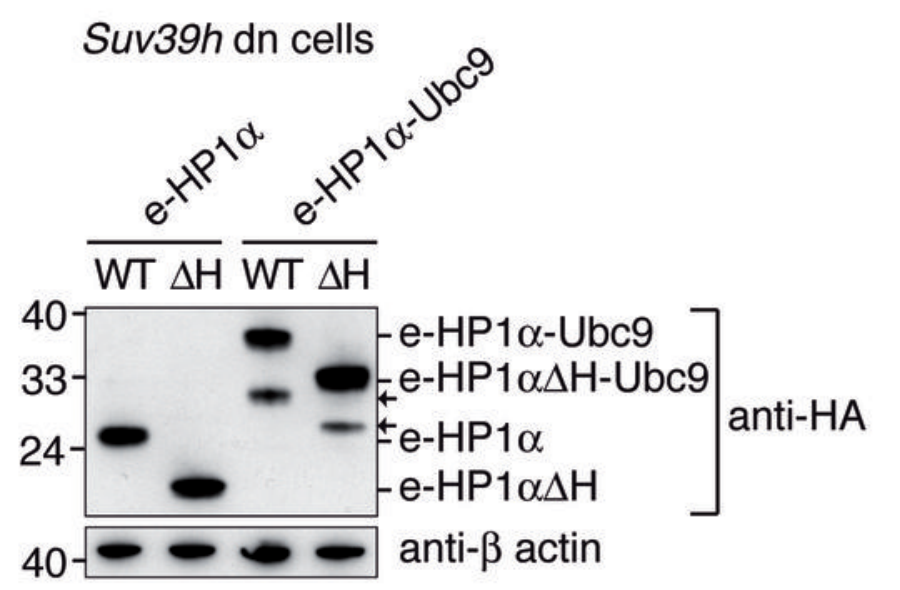

C

Suv39h dn cells

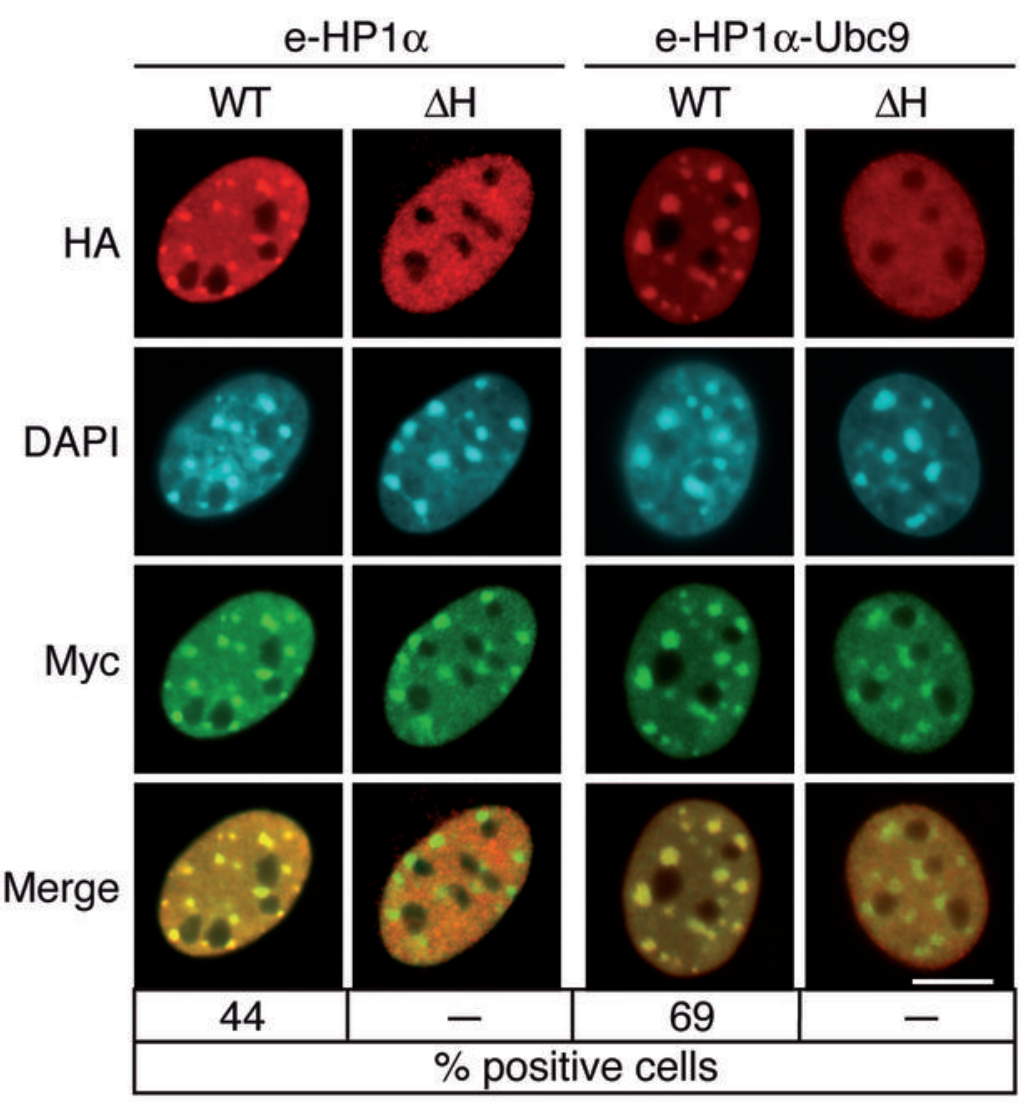

Maison et al Figure 5 


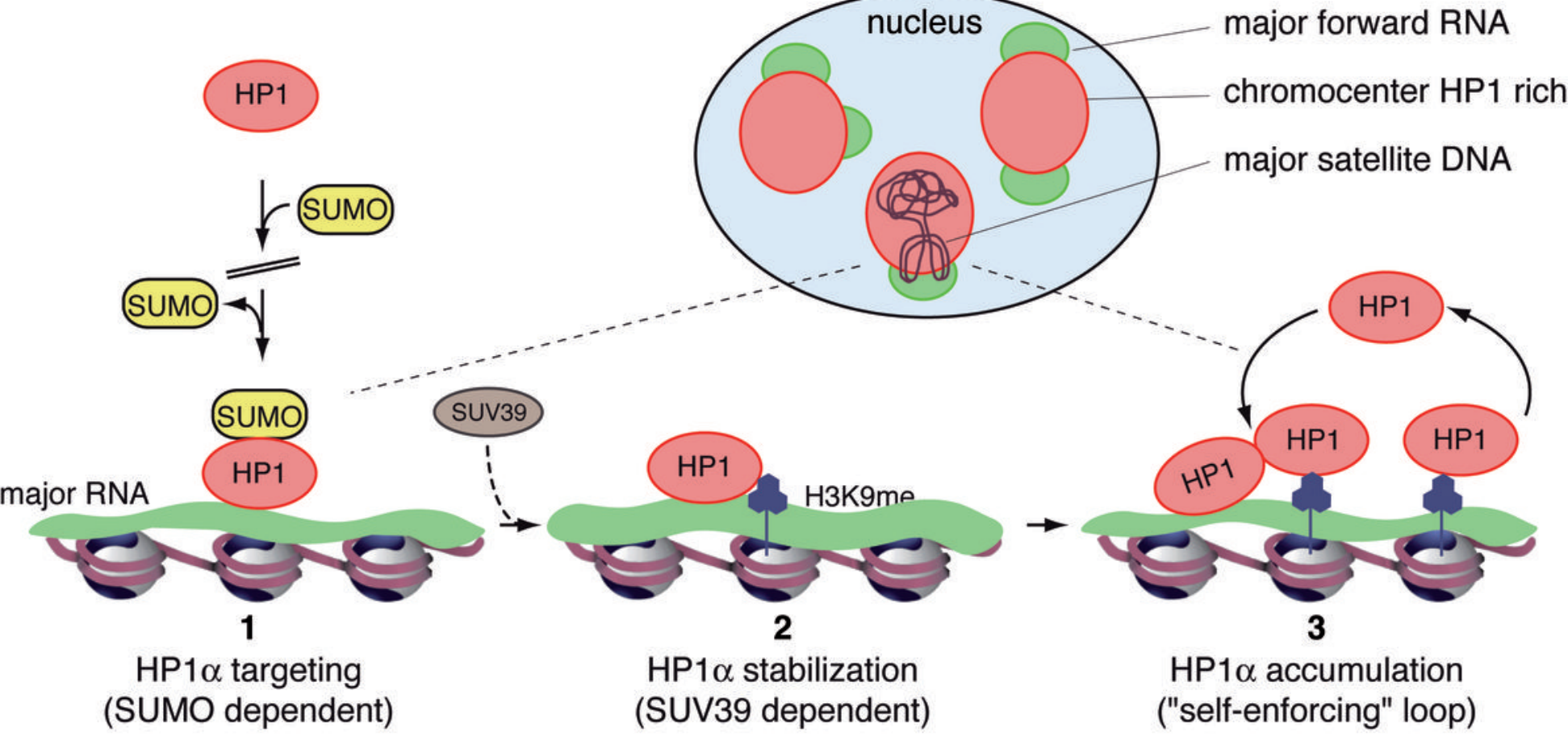

Maison et al Figure 6 


\section{SUPPLEMENTARY DATA}

SUMOylation promotes de novo targeting of HP1 $\alpha$ to pericentric heterochromatin

Christèle Maison, Delphine Bailly, Danièle Roche, Rocío Montes de Oca, Aline V. Probst, Isabelle

Vassias, Florent Dingli, Bérengère Lombard, Damarys Loew, Jean-Pierre Quivy and Geneviève Almouzni 
major RNA

forward + reverse

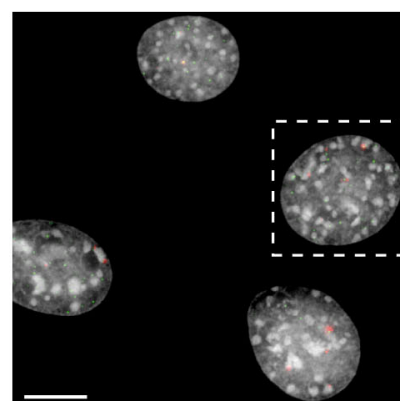

+ RNase

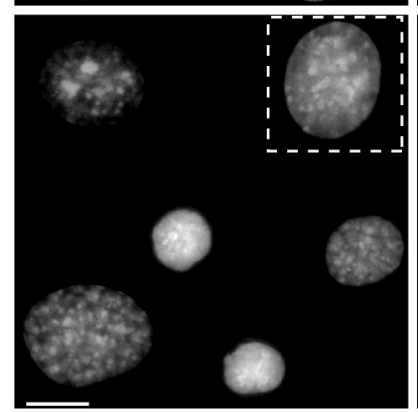

minor RNA

Enlargement
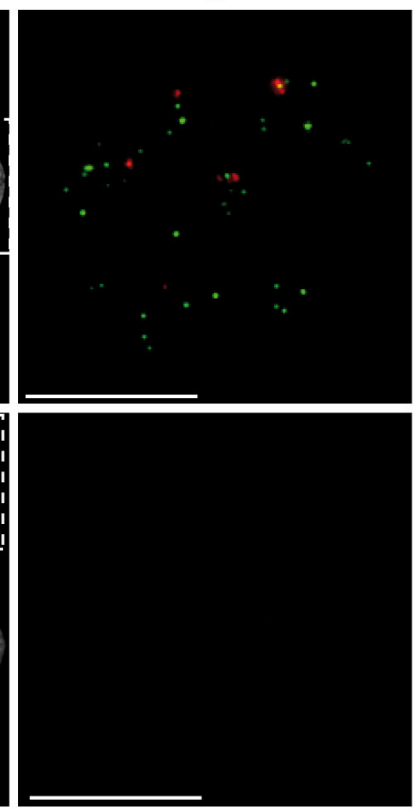

forward + reverse
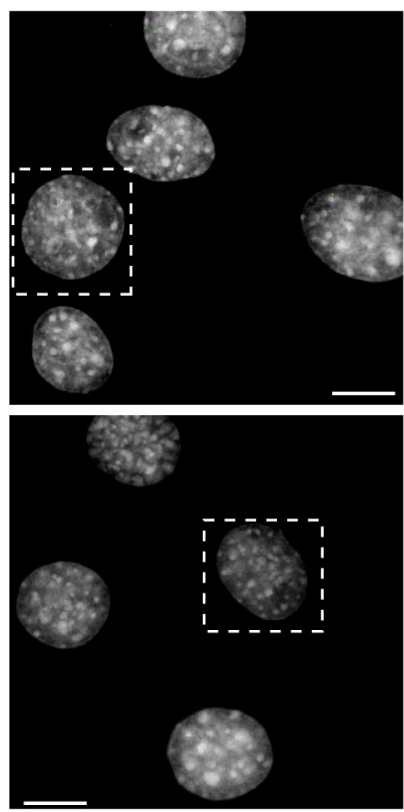
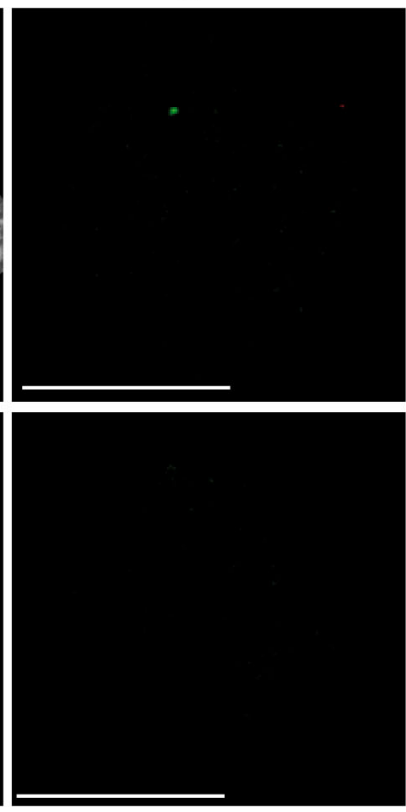

Supplementary Figure 1. Localization of major and minor transcripts. RNA FISH analysis of NIH3T3 cells mock (- RNase) or RNase (+ RNase) treated. Major (forward in green, reverse in red) and minor (forward in red, reverse in green) RNAs are detected with strand-specific LNA probes. Enlargement: magnification of the nucleus inside the white box. Scale bar, $10 \mu \mathrm{M}$. 
forward major RNA RNA and DAPI
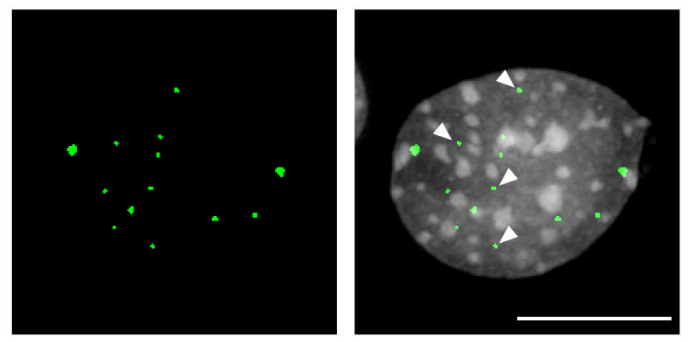

localization of forward major RNA with respect to pericentric domains

$(\%)$

\begin{tabular}{|l|l|}
\hline close & 70 \\
\hline away & 30 \\
\hline
\end{tabular}

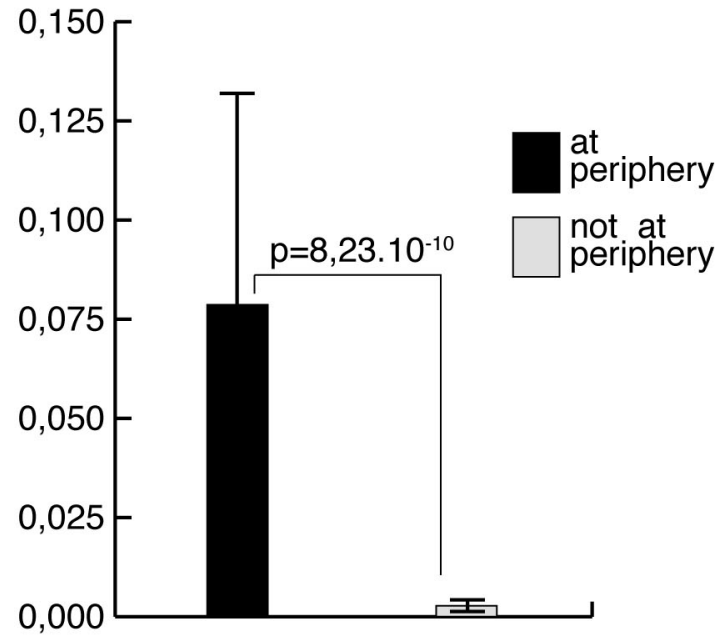

major DNA

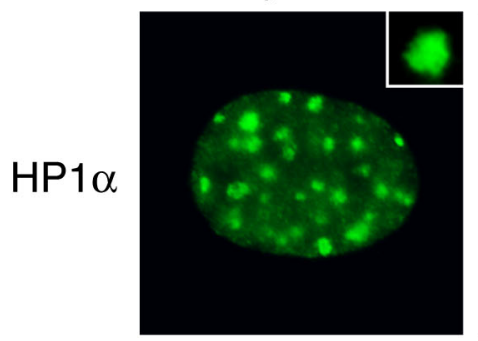

DNA
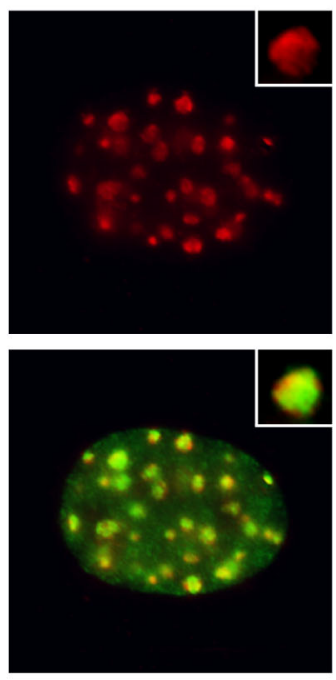

merge

DAPI

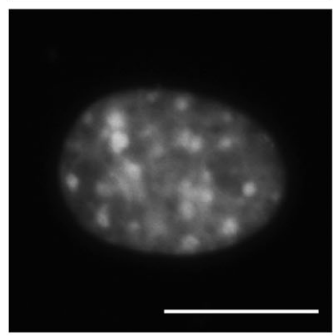

minor DNA
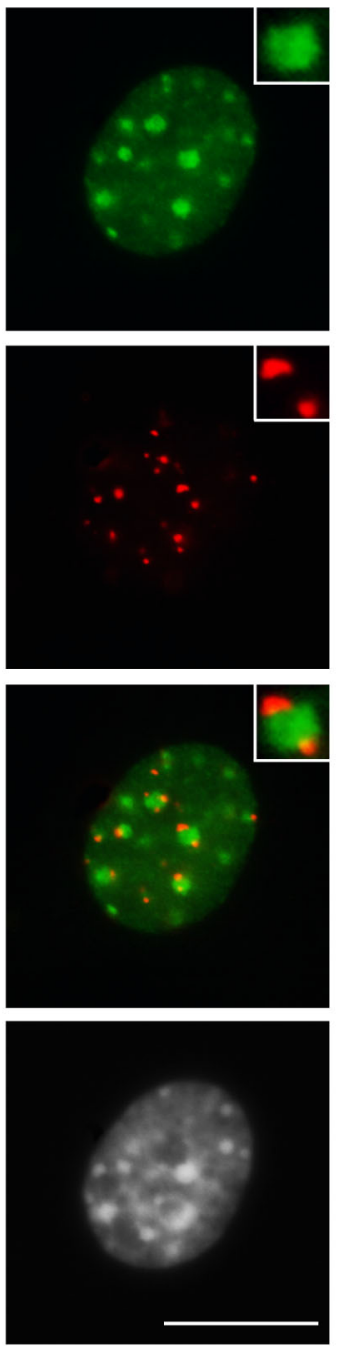

Supplementary Figure 2. Localization of major transcripts. a. Quantification of forward major RNA localization at the periphery of pericentric domains. Top : RNA FISH. We show forward major RNA (green) along with a merged image of DAPI and RNA staining. Arrowheads indicate RNA foci away from pericentic domains. Middle: we calculated from 35 nuclei the percentage of forward major RNA foci (arrowheads) localized close to and away from pericentric domains. Bottom : graph comparing the presence of forward major RNA (foci/ $\mu \mathrm{m} 3$ ) at the periphery (black) or not at the periphery (grey) of pericentric domains from 35 nuclei which represent 408 pericentric domains and 196 RNA foci in 3 independent experiments. The error barr (SD) and the $p_{\text {value }}$ are indicated. b. HP1a accumulation at major satellite DNA domains. Immuno-DNA FISH with antiHP1a antibodies (green) and major or minor satellite DNA probes (red). Insets show magnification of a chromocenter. Scale bar, $10 \mu \mathrm{M}$. 
a in vitro transcribed biotinylated-RNA
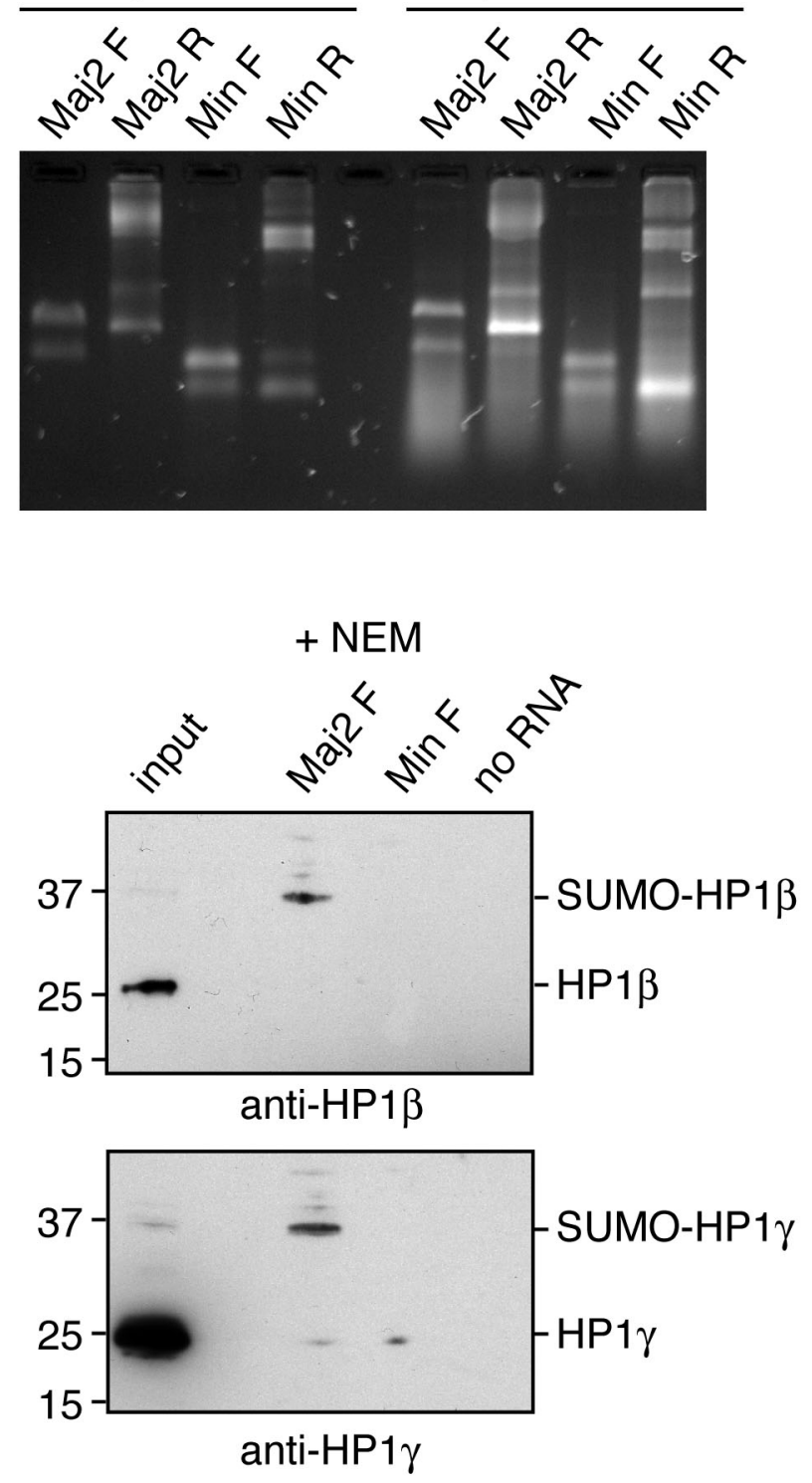

\begin{tabular}{c|c}
50 & - Ptbp 1 \\
-37 & - RBMX \\
& hnRNP A/B \\
& hnRNP C \\
& $\begin{array}{l}\text { Elav-like } \\
\text { protein 1 }\end{array}$ \\
25 &
\end{tabular}

Coomassie
hnRNP $M$

$\exists$ FUSE-BP hnRNP R NS1-associated protein 1

Supplementary Figure 3. Identification of proteins associated with centromeric RNAs. a. Ethidium bromide stained agarose gel shows in vitro transcribed biotinylated-RNAs before $(10 \%$ of starting material) and after affinity purification using forward $(F)$ and reverse $(R)$ major (Maj2 $F$, Maj2 $R$ ) or minor (Min F, Min R) RNAs as baits. b. RNA pull down using forward major (Maj2 F) or minor (Min F) RNAs as baits, in the absence of NEM. A coomassie blue stained gel is shown and selected proteins identified by mass spectrometry are indicated. Bold proteins indicate proteins specifically associated with forward major or minor RNAs. c. RNA pull down using forward major (Maj2 F) or minor (Min F) RNAs, or no RNA as negative control, in the presence of NEM. Western blot analysis using anti-HP1 $\beta$ and anti-HP1Y specific antibodies revealed endogenous SUMO-HP1 $\beta, \mathrm{HP} 1 \beta$, SUMO-HP1Y and HP1Y. Input is $10 \%$ of nuclear extracts. 
a NIH3T3 cells Transfection

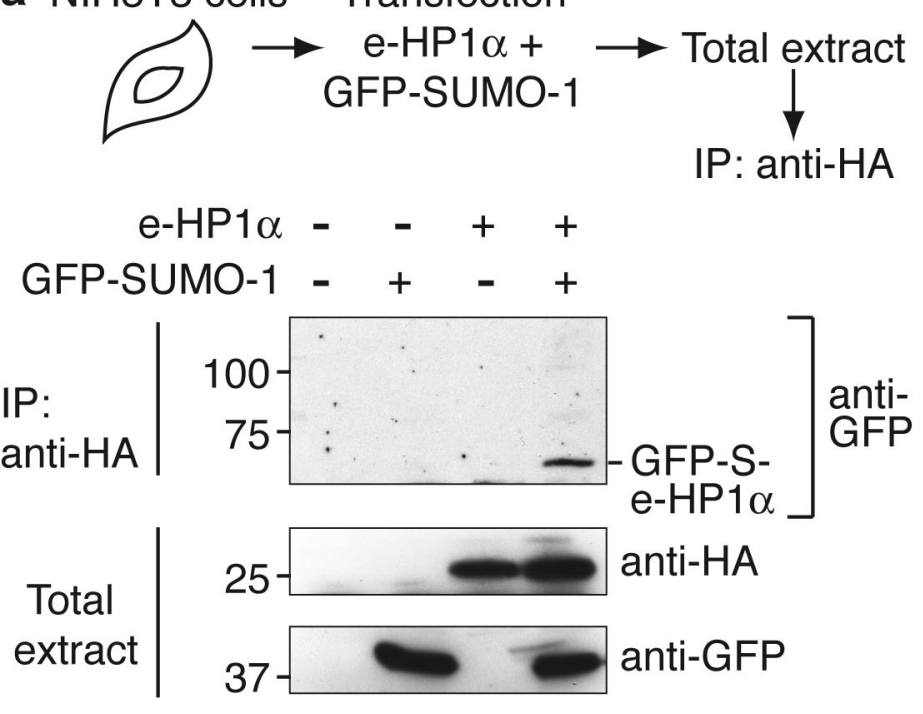

b
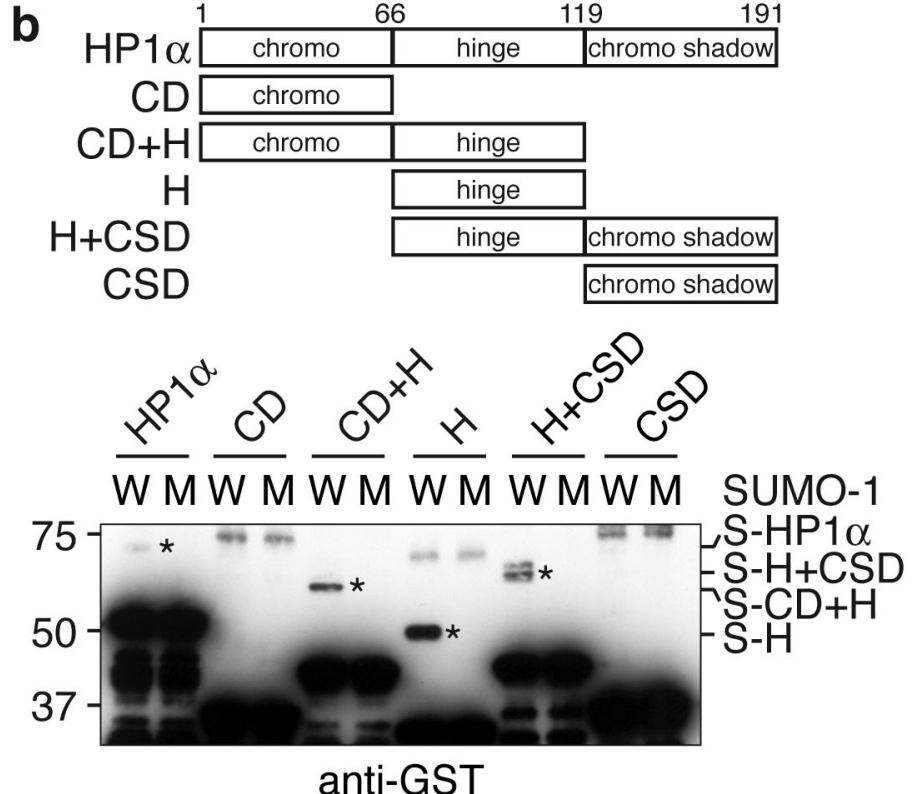
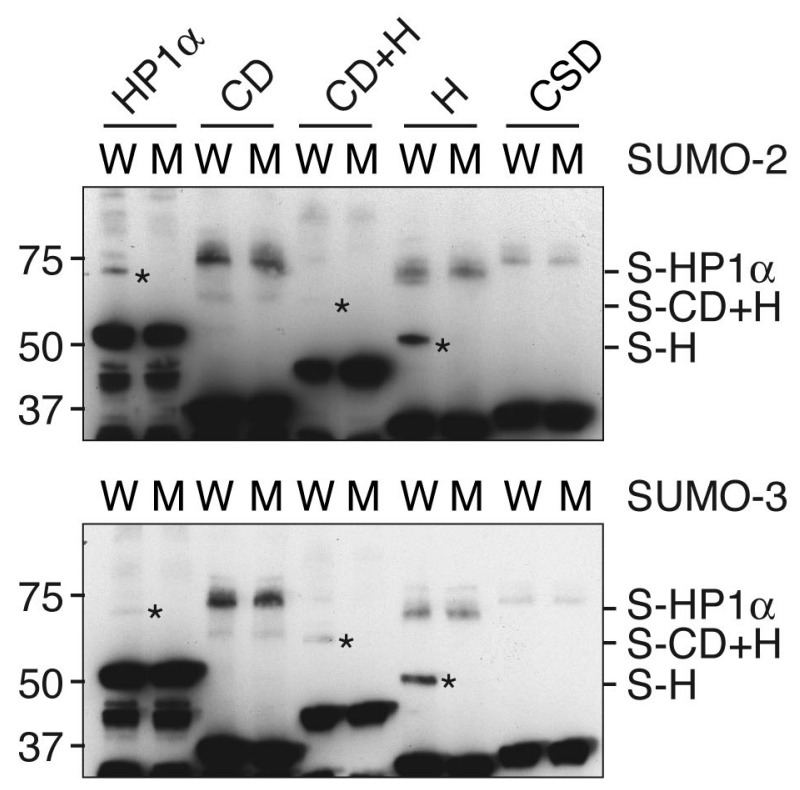

anti-GST

Supplementary Figure 4. The hinge domain of HP1a is sumoylated by SUMO-1 or SUMO-2/3. a. HP1 a sumoylation in vivo. Top: Experimental scheme. Bottom: We mock transfected or transiently transfected NIH3T3 cells with either e-HP1a, GFP-SUMO-1 or e-HP1a with GFP-SUMO-1, as indicated. $48 \mathrm{~h}$ after transfection, we lysed cells in the presence of NEM and immunoprecipitated total extracts using anti-HA beads. Western blotting using anti-GFP antibodies revealed sumoylated e-HP1a (GFP-S-e-HP1a). We verified the expression of e-HP1a and GFP-SUMO-1 in total cell extracts using anti-HA and anti-GFP antibodies. b. HP1a sumoylation in vitro. Top: Schematic representation of full-length HP1a and fragments thereof. Bottom: Western blot analysis of the SUMO-1 reaction mixtures by anti-GST antibodies revealed the positions of SUMO-1-modified HP1a full-length (S-HP1a) or fragment domains (S-H+CSD, S-CD+H and $\mathrm{S}-\mathrm{H}$ ), indicated by asterisks $\left(^{*}\right)$. c. HP1a sumoylation in vitro. Western blot analysis of the SUMO-2 (top) and SUMO-3 (bottom) reaction mixtures by anti-GST antibodies revealed the positions of sumoylated HP1a full-length (S-HP1a) or fragment domains (S-CD+H and S-H), indicated by asterisks $\left(^{*}\right)$. 
a

NIH3T3 cells
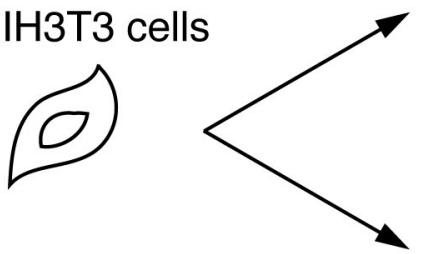

(b) Transfection

- empty vector

- e-HP1 $\alpha$

- e-HP1 $\alpha-U b c 9$

$\rightarrow$ Total extract

IP: anti-HA

Western blot

- e-Ubc9

(c) Co-transfection

+ GFP-SUMO-1

- e-HP1 $\alpha$-Ubc9wt

- e-HP1 $\alpha$-Ubc9C93S

-e-HP1 $\alpha$

IP: anti-HA

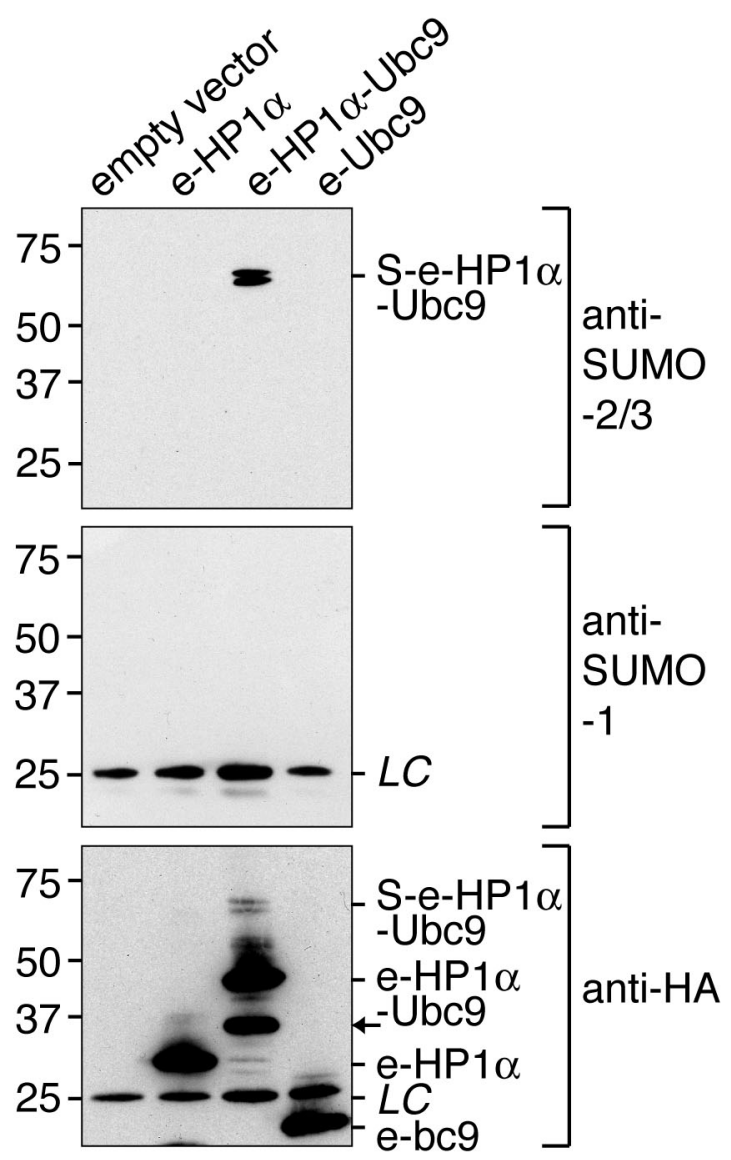

C

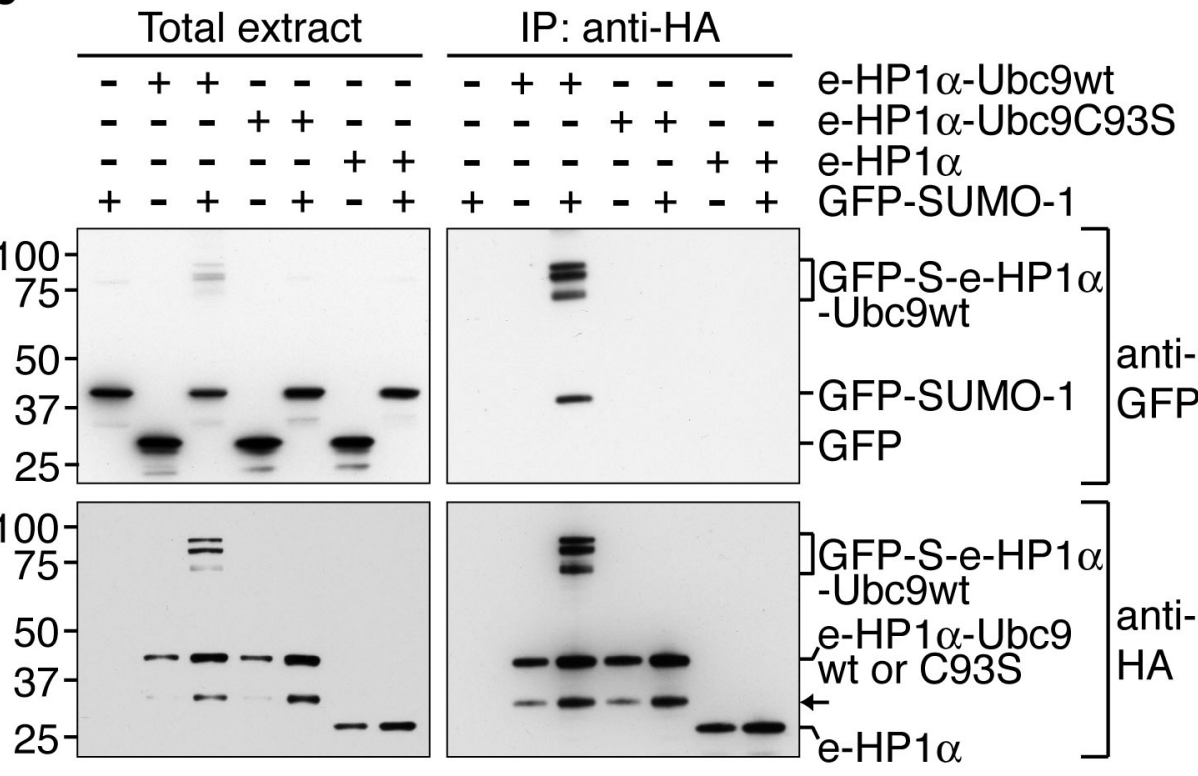

Supplementary Figure 6. HP1a-Ubc9 is modified by SUMO-1 in vivo. a. Experimental scheme. b. HP1 $\mathrm{a}-U \mathrm{bc} 9$ sumoylation in vivo. $48 \mathrm{~h}$ after transfection of NIH3T3 cells with either an empty vector, e-HP1a, e-HP1a-Ubc9 or e-Ubc9, we lysed cells in the presence of NEM and immunoprecipitated total extracts using anti-HA beads. Western blotting using anti-SUMO-2/3, anti-SUMO-1 and anti-HA antibodies revealed the positions of SUMO-modified e-HP1a-Ubc9 (S-e-HP1a-Ubc9), unmodified e-HP1a-Ubc9, e-HP1a or e-Ubc9. LC corresponds to the immunoglobulin light chain. Arrow indicates a degradation product of e-HP1 a-Ubc9. c. HP1a-Ubc9 sumoylation in vivo. We transfected NIH3T3 cells with e-HP1a-Ubc9wt, e-HP1 a-Ubc9C93S, e-HP1a alone or with GFP-SUMO-1, as indicated. We verified the expression of the proteins in total cell extracts using anti-GFP and anti-HA antibodies (left). In immunoprecipitates, Western blotting using anti-GFP revealed sumoylated e-HP1a-Ubc9wt (GFP-S-e-HP1a-Ubc9wt) and using anti-HA antibodies revealed sumoylated e-HP1a-Ubc9wt (GFP-S-e-HP1a-Ubc9wt) and unmodified e-HP1a-Ubc9wt, e-HP1a-Ubc9C93S, e-HP1a (right). Arrow indicates a degradation product of e-HP1a-Ubc9. 

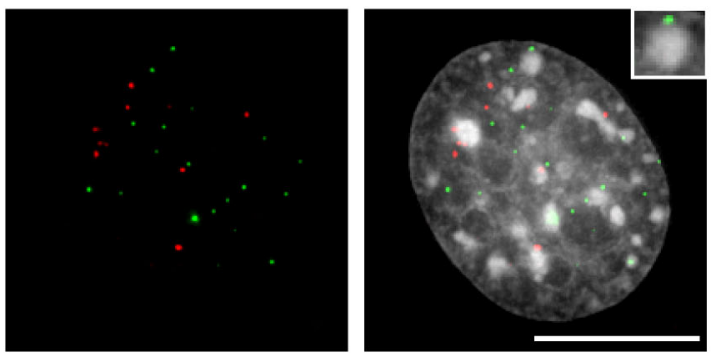

b

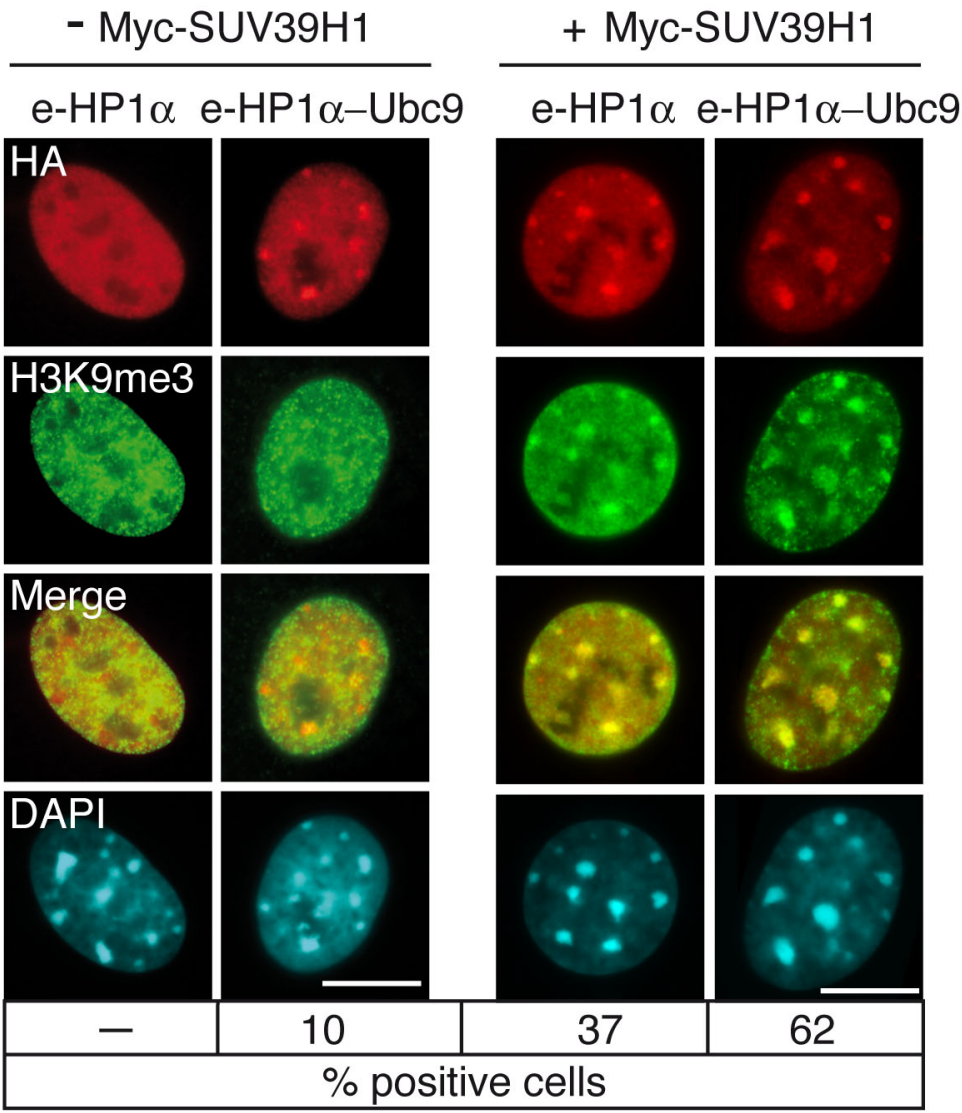

C - Myc-SUV39H1

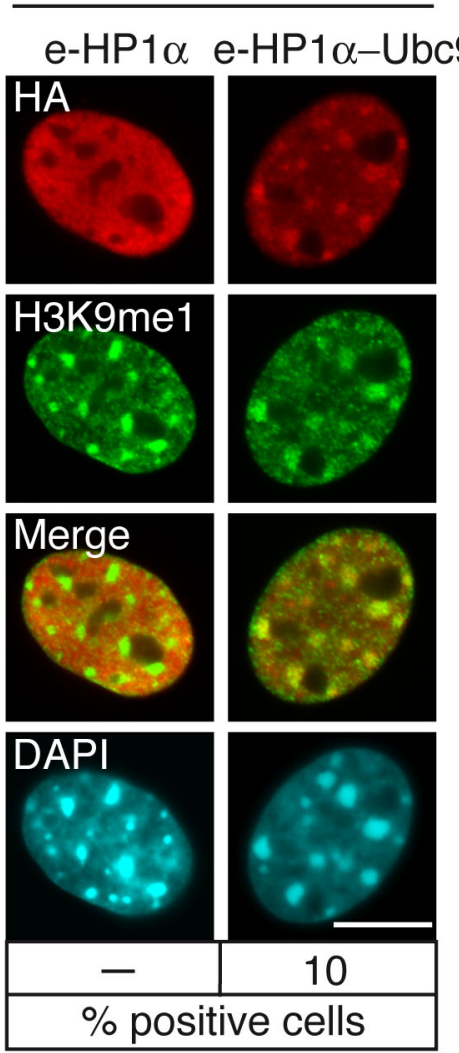

Supplementary Figure 7. HP1a-Ubc9 is targeted to pericentric heterochromatin in the absence of H3K9me3. a. Localization of major RNA in Suv39h double-null cells by RNA FISH. Left: forward (green) and reverse (red) major RNAs detected with strand-specific LNA probes. Right: merged image of DAPI and RNA staining. The inset shows a magnification of a chromocenter. Scale bar, $10 \mu \mathrm{M}$. b. Localisation of e-HP1a and e-HP1a-Ubc9 in the absence (left) or in the presence (right) of Myc-SUV39H1 in Suv39h dn cells by immunofluorescence using anti-HA (red) and anti-H3K9me3 (green) antibodies $6 \mathrm{~h}$ after transfection. For each condition, we examined 300 transfected cells and calculated the percentage of cells with $\mathrm{HA}$ signal enriched at pericentric domains (positive cells). Scale bar, $10 \mu \mathrm{M}$. c. Localization of e-HP1a and e-HP1a-Ubc9 in Suv39h dn cells by immunoflorescence using anti-HA (red) and anti-H3K9me1 (green) antibodies $6 \mathrm{~h}$ after transfection. The percentage of positive cells was calculated as in b. The Suv39h dn cells showed H3K9me1 staining at pericentric domains. Scale bar, $10 \mu \mathrm{M}$. 


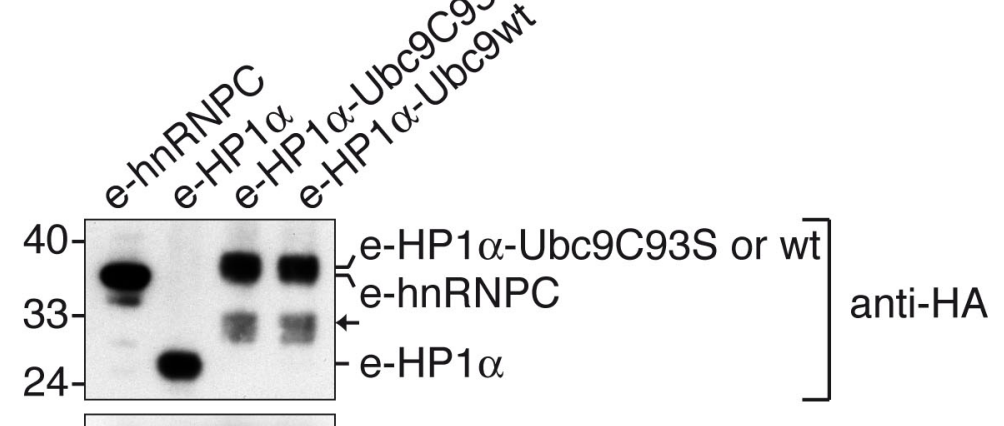

$40-00$ anti- $\beta$ actin

b

- Triton

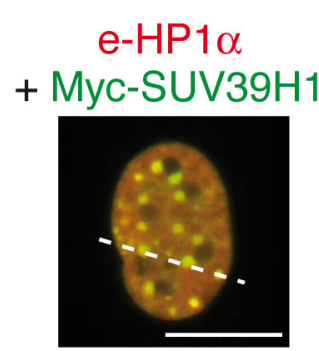

merge

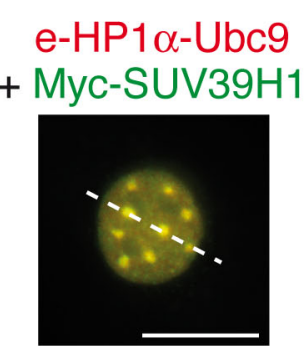

merge
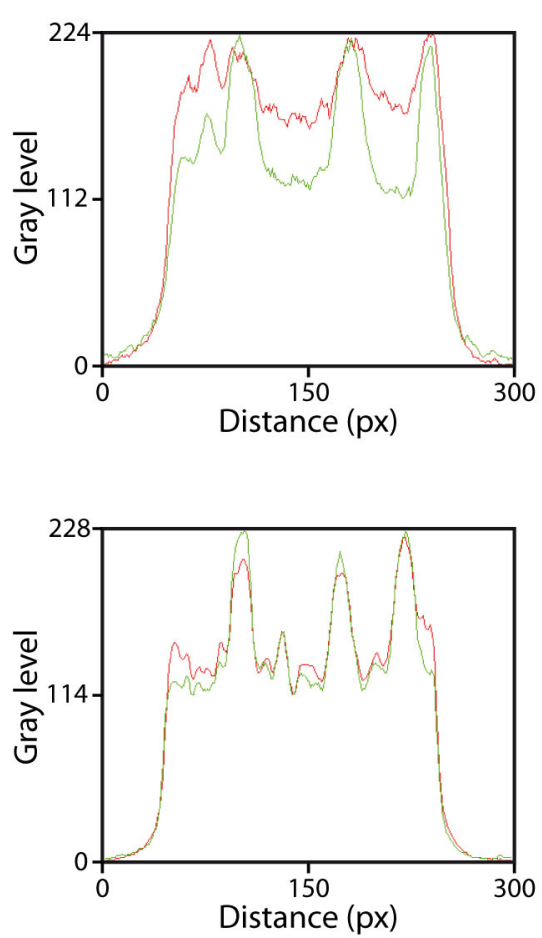

+ Triton

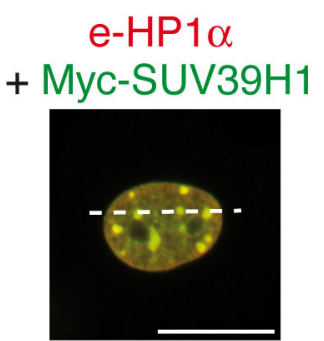

merge

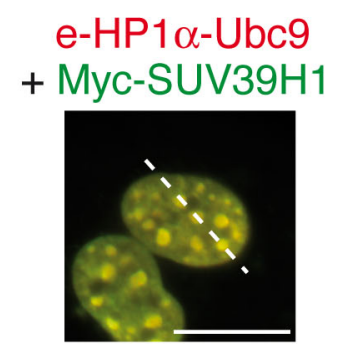

merge
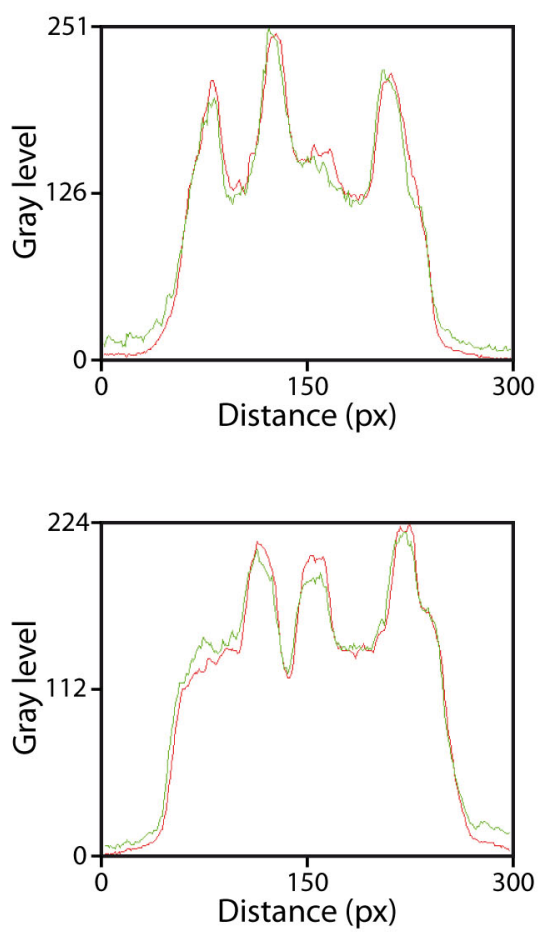

Supplementary Figure 8. Sumoylation of HP1a promotes its accumulation at pericentric heterochromatin. a. Comparison of protein expression corresponding to the experiment showed in Fig. 4e by Western blot using anti-HA and anti- $\beta$ actin antibodies. Arrow indicates a degradation product of e-HP1 a-Ubc9. b. We co-transfected Suv39h dn cells with Myc-SUV39H1 and either e-HP1a or e-HP1a-Ubc9, and performed immunofluorescence with anti-HA (red) and anti-Myc (green) antibodies in untreated (left, - Triton) and Triton extracted (right, + Triton) cells. For each condition, a merge image of Myc and $\mathrm{HA}$ staining is shown with a line scan presenting the relative intensities of the Myc and HA signals across the nucleus. Representation of intensity (gray level) vs distance (pixels) is shown. Scale bar, $10 \mu \mathrm{M}$. Using this line scan analysis, we confirmed that while e-HP1a-Ubc9 and Myc-SUV39H1 overlapped in the absence of Triton treatment (left), e-HP1a showed a diffuse staining that spread throughout the nucleus. To determine if this diffuse staining was not due to ectopic localization at many sites within chromatin, we examined HP1a localization after Triton extraction (right). Under these conditions, the diffuse staining was no longer observed and the HP1a chromatin-bound dots showed a perfect overlap with SUV39H1 domains. 


\section{a Suv39h dn cells}

Co-transfection $\mid \cdot \mathrm{e}-\mathrm{HP} 1 \alpha$

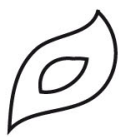

$\rightarrow+$ Myc-SUV39H1 $+\begin{aligned} & \cdot \text { e-HP1 } \alpha-S U M O-1 \\ & \cdot e-H P 1 \alpha-U b c 9\end{aligned}$ (b) de novo localization assay after $4 \mathrm{~h}$

(c) cell viability

\section{b e-HP1 $\alpha$ e-HP1 $\alpha-S U M O$}

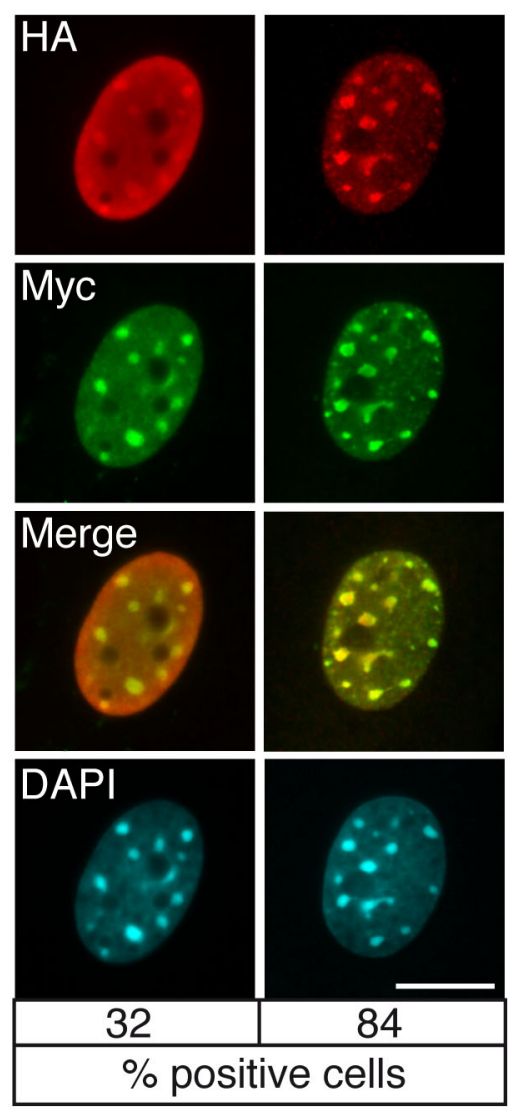

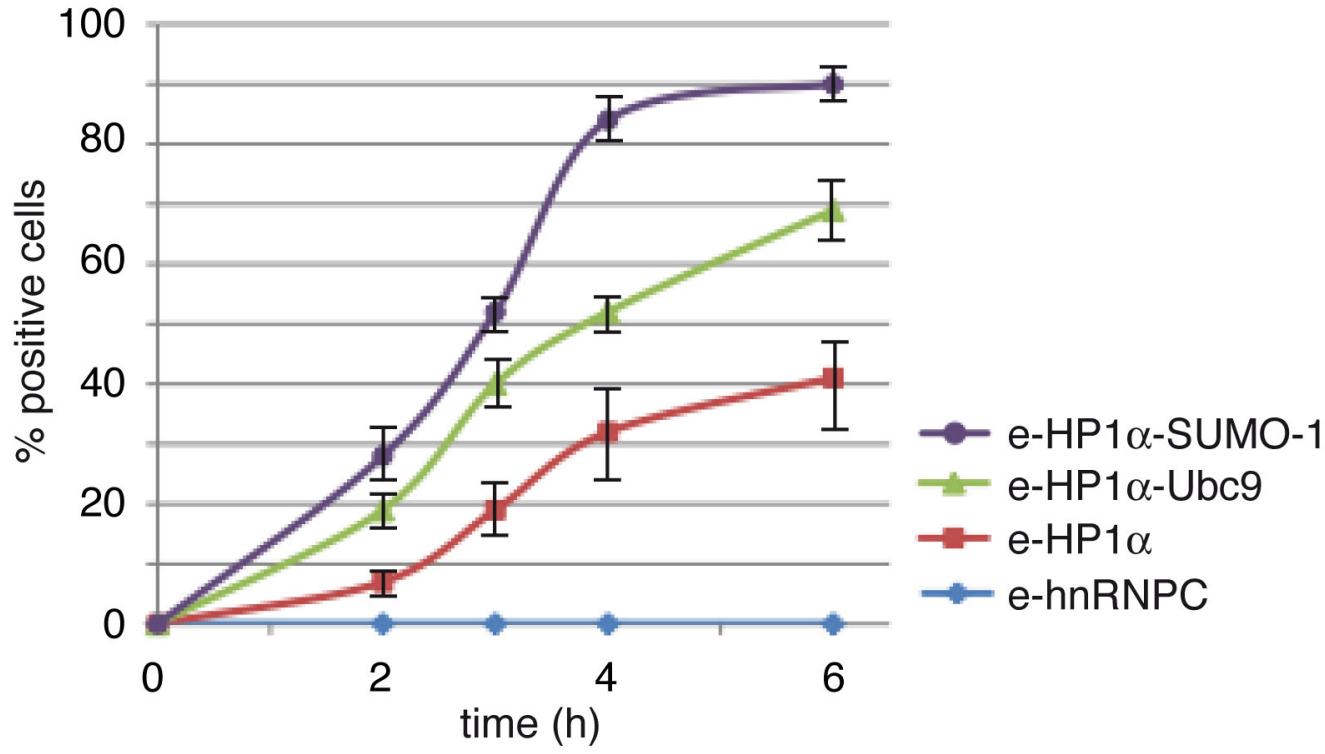

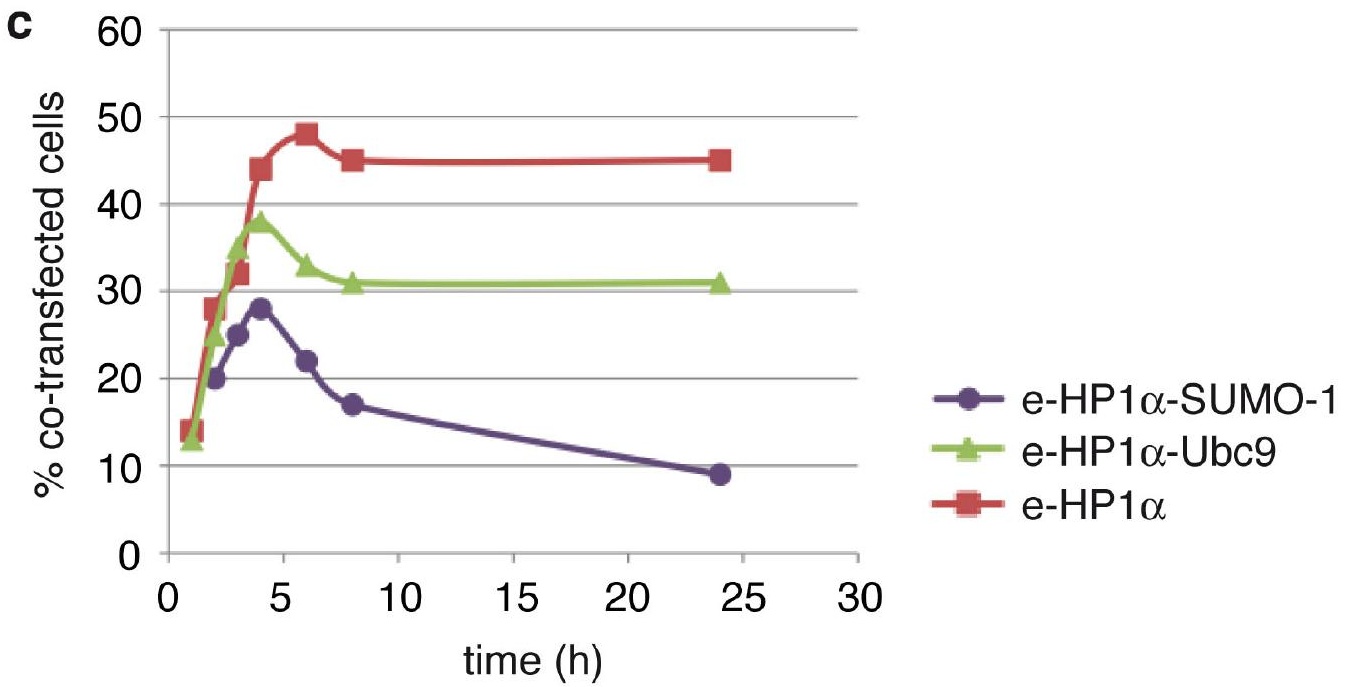

Supplementary Figure 9. HP1a-SUMO-1 accumulates at pericentric domains. a. Experimental scheme. We tested whether fusing directly SUMO-1 to HP1a could suffice to promote its efficient localization at pericentric domains. After co-transfection of an e-HP1a-SUMO-1 fusion construct together with Myc-SUV39H1 in Suv39h dn cells, we found that e-HP1a-SUMO-1 accumulated $\sim 2.5$-fold more efficiently to pericentric domains compared to e-HP1a (b). However, only $9 \%$ of cells co-transfected with e-HP1 a-SUMO-1 were viable $24 \mathrm{~h}$ after transfection compared to $45 \%$ and $31 \%$ of e-HP1a and e-HP1a-Ubc9, respectively (c). Given the high mortality in this assay, we decided to carry out further experiments with HP1a-Ubc9 which proved less toxic. b. Left: de novo localization of e-HP1a and e-HP1a-SUMO-1 in Suv39h dn cells co-transfected with Myc-SUV39H1 by immunofluorescence using anti-HA (red) and antiMyc (green) antibodies $4 \mathrm{~h}$ after transfection. For each condition, we examined 300 co-transfected cells and calculated the percentage of cells with HA signal enriched at pericentric domains (positive cells). Scale bar, $10 \mu \mathrm{M}$. Right: Time course analysis of the de novo localization of e-HP1a , e-HP1a-Ubc9, e-HP1a-SUMO-1 and e-hnRNPC in Suv39h dn cells co-transfected with Myc-SUV39H1. The percentage of positive cells as a function of the time after transfection is represented. Symbols indicate the mean and error bars indicate the standard deviation of three independent experiments (300 co-transfected cells counted in each condition). c. Cell viability after co-transfection of Suv39h dn cells with Myc-SUV39H1 and either e-HP1a, e-HP1a-Ubc9 or e-HP1a-SUMO-1. After immunofluorescence with anti-HA and antiMyc antibodies, we examined 300 cells per condition and represented the number of co-transfected cells as a percentage of the number of total cells, and as a function of the time after transfection. 


\begin{tabular}{|c|c|c|c|c|c|c|}
\hline \multicolumn{7}{|c|}{ Major RNA interacting proteins } \\
\hline Identifier & Protein name & Description & MW (Da) & $\begin{array}{l}\text { Mascot } \\
\text { score }\end{array}$ & Peptides & $\begin{array}{c}\text { Coverage } \\
(\%)\end{array}$ \\
\hline gil19527028 & Vigilin & high density lipoprotein binding protein & 141743 & 675.79 & 14 & 14.1 \\
\hline gil21450287 & UNR & DNA segment, Chr 3, MJeffers 1 & 88790.6 & 180.36 & 4 & 5.1 \\
\hline gil21313308 & hnRNP M & heterogeneous nuclear ribonucleoprotein $\mathrm{M}$ & 77648.7 & 254.84 & 5 & 8.0 \\
\hline gil13242328 & NS1-associated protein 1 & NS1-associated protein 1 & 69769.8 & 419.34 & 8 & 10.7 \\
\hline gil51263 & p68 RNA helicase & p68 RNA helicase & 69320.3 & 118.47 & 3 & 5.9 \\
\hline gil7305075 & G3bp & $\begin{array}{l}\text { ras-GTPase-activating protein SH3-domain } \\
\text { binding protein }\end{array}$ & 51828.8 & 128.79 & 3 & 9.9 \\
\hline gil6755296 & RBMX & RNA binding motif protein, $\mathrm{X}$-linked & 42300.9 & 308.13 & 5 & 14.8 \\
\hline
\end{tabular}

\begin{tabular}{|c|c|c|c|c|c|c|}
\hline \multicolumn{7}{|c|}{ Minor RNA interacting proteins } \\
\hline Identifier & Protein name & Description & MW (Da) & $\begin{array}{l}\text { Mascot } \\
\text { score }\end{array}$ & Peptides & $\begin{array}{c}\text { Coverage } \\
(\%)\end{array}$ \\
\hline gil19527028 & Vigilin & high density lipoprotein binding protein & 141743 & 89.67 & 2 & 1.2 \\
\hline gil17390825 & hnRNP U & Hnrpu protein & 87917.7 & 136.22 & 2 & 4.1 \\
\hline gil21313308 & hnRNP M & heterogeneous nuclear ribonucleoprotein $\mathrm{M}$ & 77648.7 & 39.34 & 1 & 1.4 \\
\hline gil13242328 & NS1-associated protein 1 & NS1-associated protein 1 & 69769.8 & 132.22 & 3 & 4.5 \\
\hline gil13938631 & Ptbp 1 & Ptbp1 protein & 59321.6 & 218.72 & 6 & 18.4 \\
\hline gil6755296 & RBMX & RNA binding motif protein, $\mathrm{X}$-linked & 42300.9 & 116.85 & 2 & 6.9 \\
\hline gil6754222 & hnRNP A/B & heterogeneous nuclear ribonucleoprotein $A / B$ & 30831.3 & 81.89 & 3 & 9.1 \\
\hline gil8393544 & hnRNP C & heterogeneous nuclear ribonucleoprotein C & 34384.8 & 173.06 & 4 & 12.8 \\
\hline
\end{tabular}

\footnotetext{
Supplementary Table 1. List of selected major and minor RNA associated proteins identified by mass spectrometry. Table shows identifier (NCBI protein accession number), description (NCBI protein definition), MW (molecular weight in Daltons), mascot score (sum of the unique mascot ions scores), peptides (number of peptides identified per experiment), coverage (\% of sequence coverage identified from MS/MS data). Protein name in bold indicates proteins that associated with only major or minor RNAs. We performed the experiment twice and showed the best experiment.
} 
Supplementary Table 2. Probe and primer sequences

LNA fluorescent probes for RNA and DNA FISH

\begin{tabular}{|l|l|l|}
\hline Name & Fluorophore & Sequence \\
\hline major 1 & FITC & TCTTGCCATATTCCACGTCC \\
major 2 & Cy3 & GCGAGGAAAACTGAAAAAGG \\
minor 1 & Cy3 & GTTCTACAATGCCGGTTTCC \\
minor 2 & FITC & TACACTGAAAAACACATTCG \\
\hline
\end{tabular}

\section{Primers for RT-PCR}

\begin{tabular}{|l|l|}
\hline Name & Sequence (5'- 3') \\
\hline major satellites (For) & AAATACACACTTTAGGACG \\
major satellites (Rev) & TCAAGTGGATGTTTCTCATT \\
minor satellites (For) & GAAAATGATAAAAACCACAC \\
minor satellites (Rev) & ACTCATTGATATACACTGTT \\
\hline
\end{tabular}

